\title{
1 An activity-based labelling method for the detection of ammonia and 2 methane-oxidizing bacteria
}

5 Dimitra Sakoula ${ }^{1 \#^{*}}$, Garrett J. Smith ${ }^{1}$, Jeroen Frank ${ }^{1,2}$, Rob J. Mesman ${ }^{1}$, Linnea F.M. Kop ${ }^{1}$, Mike 6 S.M. Jetten ${ }^{1,2}$, Maartje A.H.J. van Kessel ${ }^{1}$, Sebastian Lücker ${ }^{1 *}$.

10 Netherlands.

11 2Soehngen Institute of Anaerobic Microbiology, Radboud University, Heyendaalseweg 135, 6525 AJ Nijmegen, 12 the Netherlands.

13 \#Present address: Division of Microbial Ecology, Center for Microbiology and Environmental Systems Science,

14 University of Vienna, Althanstraße 14, 1090, Vienna, Austria.

15

$16 *$ Corresponding authors:

18 Dimitra Sakoula, Division of Microbial Ecology, Center for Microbiology and Environmental Systems Science,

19 University of Vienna, Althanstraße 14, 1090, Vienna, Austria; phone: +43 14277 91201, mail:

20 dimitra.sakoula@univie.ac.at

22 Sebastian Lücker, Department of Microbiology, IWWR, Radboud University, Heyendaalseweg 135, 6525 AJ

23 Nijmegen, the Netherlands; phone: +31 24 3652618, mail: s.luecker@science.ru.nl. 
27 The authors declare no conflicts of interest.

\section{Abstract}

30 The advance of metagenomics in combination with intricate cultivation approaches has facilitated the

31 discovery of novel ammonia- and methane-oxidizing microorganisms, indicating that our understanding

32 of the microbial biodiversity within the biogeochemical nitrogen and carbon cycles still is incomplete.

33 However, the in situ detection and phylogenetic identification of novel ammonia- and methane-oxidizing

34 bacteria remains a challenge. Here, we describe an activity-based protein profiling protocol allowing 35 cultivation-independent unveiling of ammonia- and methane-oxidizing bacteria. In this protocol, 1,7-

36 octadiyne is used as a bifunctional enzyme probe that, in combination with a highly specific alkyne-azide

37 cycloaddition reaction, enables the fluorescent or biotin labelling of cells harboring active ammonia and

38 methane monooxygenases. The biotinylation of these enzymes in combination with immunogold labelling

39 reveals the subcellular localization of the tagged proteins, while the fluorescent labelling of cells harboring

40 active ammonia or methane monooxygenases provides a direct link of these functional lifestyles to

41 phylogenetic identification when combined with fluorescence in situ hybridization. Furthermore, we show

42 that this activity-based labelling protocol can be successfully coupled with fluorescence-activated cell

43 sorting for the enrichment of nitrifiers and methanotrophs from complex environmental samples,

44 facilitating the retrieval of their high quality metagenome-assembled genomes. In conclusion, this study

45 demonstrates a novel, functional tagging technique for the reliable detection, identification, and

46 enrichment of ammonia- and methane-oxidizing bacteria present in complex microbial communities. 


\section{Introduction}

60 Over the last two decades, metagenomic approaches resulted in the identification of novel groups of

61 ammonia- (1-3) and methane-oxidizing microorganisms (4-6), highlighting that our understanding of the 62 microbial biodiversity within the nitrogen and carbon biogeochemical cycles can still be expanded (7).

63 However, even when detected in metagenomic datasets, linking a function to a specific microorganism

64 remains challenging, as it often requires tedious and intricate cultivation techniques to isolate these often

65 slow-growing and fastidious microorganisms. Thus, there is an urgent need for robust cultivation-

66 independent methods that provide reliable information regarding the identity and activity of the

67 microorganisms present in complex microbial communities. To achieve this, various in vivo and in vitro

68 activity-based protein profiling (ABPP) protocols have been developed to link specific functions to

69 catalytically active proteins (8).

70 ABPP techniques employ bifunctional enzyme probes that feature (i) a reactive group, which binds to the 71 active site and thereby inhibits and covalently labels the enzyme, and (ii) an ethynyl or azide group, which

72 can subsequently be used for the attachment of a reporter group (e.g., fluorophores or biotin) to the 73 enzyme via a $\mathrm{Cu}(\mathrm{I})$-catalyzed alkyne-azide cycloaddition (CuAAC) reaction (9). The use of rapid CuAAC 74 reactions performed under mild, aqueous conditions guarantees minimal unspecific reactivity of the 75 reporters and enables the use of low molecular weight bifunctional probes that can easily penetrate 76 biological membranes (10-12). Subsequently, depending on the reporter type, the reporter-conjugated 77 enzyme can be subjected in numerous downstream applications such as fluorescent imaging (13-15), mass 78 spectrometry-based proteomics $(16,17)$ and affinity purification of the labelled proteins $(18)$. Several 79 microbial proteins have been studied using ABPP protocols so far. These studies provided significant 80 insights into antibiotic resistance, enzymatic functions in pathogenic bacteria (e.g., serine proteases, 81 kinases, ATPases, fatty acid synthases, glycoside hydrolases) and protein redox dynamics (8). However, 82 only a limited number of ABPP protocols targeting microbial proteins catalyzing key processes of 83 biogeochemical cycles have been developed $(2,19-21)$.

84 Autotrophic ammonia- and methane-oxidizing bacteria ( $\mathrm{OOB}$ and $\mathrm{MOB}$, respectively) (22) are ubiquitous 85 in the environment (4) and are of high biotechnological interest (23-29). Besides their similar 86 environmental niches, they share many biochemical, morphological and physiological characteristics (30).

87 More specifically, both microbial guilds can perform aerobic oxidation of ammonia and methane due to 88 the substrate promiscuity of their key enzymes, but neither group exhibits growth on the alternative 89 substrate (30-32). 
90 The ammonia monooxygenase (AMO) belongs to the protein family of copper-containing membrane-

91 bound monooxygenases and is responsible for the first oxidation step of ammonia to hydroxylamine in $92 \mathrm{AOB}$ and ammonia-oxidizing archaea (AOA). Similarly, the two different forms of methane 93 monooxygenases (MMOs) identified in $\mathrm{MOB}$ can oxidize methane to methanol (33, 34). The membrane-

94 bound particulate $\mathrm{MMO}$ ( $\mathrm{PMMO}$ ) exhibits high genetic, structural and catalytic similarities to AMO 95 enzymes (35-37) and is present in most MOB. However, some MOB also contain a cytoplasmic iron96 containing soluble $\mathrm{MMO}$ (sMMO), which is expressed mainly under copper-limited conditions $(38,39)$ and 97 can even constitute the sole $\mathrm{MMO}$ in some $\mathrm{MOB}(40)$. Although the substrate for both MMOs is identical, 98 the sMMO shares little structural homology with the pMMO and AMO enzymes (37). In recent years, some 99 advances have been made in studying these enzymes $(33,41)$. However, the recent discovery of the novel 100 comammox (complete ammonia oxidation) Nitrospira, featuring a distinct AMO enzyme previously 101 misclassified as an "unusual" pMMO $(1,2)$, exemplified our still incomplete understanding of the microbial 102 diversity catalyzing biological ammonia and methane oxidation.

103 To date, many insights into the distribution, abundance and activity of ammonia-oxidizing prokaryotes 104 (AOP) and $\mathrm{MOB}$ in natural environments have been obtained by employing reversible (42-45) and 105 irreversible inhibitors $(19,46-48)$. Many terminal $n$-alkynes can be catalytically activated by AMO and 106 MMOs, producing reactive intermediates that bind covalently to the enzyme and act as mechanism-based 107 irreversible inactivators $(47,49-52)$. The alkyne 1,7-octadiyne (1,7OD) was recently characterized as a 108 mechanism-based inactivator of the AMO enzyme in Nitrosomonas europaea and was successfully used 109 in in vitro ABPP protocols (19).

110 In this study, we describe the use of 1,70D in an ABPP-based protocol for the in situ biotin or fluorescent 111 labelling of bacterial AMO and MMOs. In combination with transmission electron microscopy, the biotin 112 tagging of the enzymes permits the study of their intracellular distribution, while their fluorescent tagging 113 allows the function-based detection of catalytically active ammonia and methane-oxidizing 114 microorganisms by fluorescence microscopy and can be combined with fluorescence in situ hybridization 115 (FISH) for phylogenetic identification. Moreover, this method can be efficiently combined with 116 fluorescence-activated cell sorting for the enrichment of ammonia- and methane-oxidizing bacteria from 117 complex environmental samples. Combined with downstream metagenomics, this facilitates the targeted 118 retrieval of high quality metagenome-assembled genomes (MAGs) of a functionally-defined 119 subpopulation.

\section{Materials and methods}




\section{Cultivation}

123 A pure culture of the canonical ammonia-oxidizing bacterium Nitrosomonas europaea (ATCC 25978) was

124 obtained from the Leibniz Institute DSMZ-German Collection of Microorganisms and Cell Cultures

125 (Braunschweig, Germany). N. europaea was grown in batch in mineral salts medium amended with 10

$126 \mathrm{mM} \mathrm{NH}_{4} \mathrm{Cl}$ (53). Cultures of the methane-oxidizing bacteria Methylotetracoccus oryzae (nitrogen-fixing,

127 Type Ib methanotroph), Methylosinus sporium M29 (Type II methanotroph), Methylacidiphilum

128 fumariolicum (Type III methanotroph) and Methylocella tundrae (Type II methanotroph containing sMMO

129 only) were maintained in batch in mineral salts medium as previously described $(6,54-56)$. A pure culture

130 of the canonical nitrite-oxidizing bacterium Nitrospira moscoviensis M-1 (DSM 10035) was grown in a

131 mineral salts medium according to Mundiger et al. (57). Nitrospira inopinata cells were grown as described

132 elsewhere in the presence of $1 \mathrm{mM} \mathrm{NH}_{4} \mathrm{Cl}$ (58). A pure culture of the diazotrophic Kyrpidia spormannii

133 FAVT5 was cultivated according to Hogendoorn et al. (59). An enrichment culture containing the

134 comammox species $\mathrm{Ca}$. Nitrospira nitrosa and $\mathrm{Ca}$. Nitrospira nitrificans was maintained in a sequencing

135 batch bioreactor as reported previously (2). An enrichment culture of the novel comammox species $\mathrm{Ca}$.

136 Nitrospira kreftii was maintained in a membrane chemostat as described elsewhere (60). A nitrifying

137 enrichment culture containing canonical AOB, canonical (i.e., lacking AMO) and comammox Nitrospira, as

138 well as non-nitrifying microorganisms was maintained in a continuous membrane bioreactor in mineral

139 salts medium under substrate-limited conditions. Activated sludge samples were obtained from the

140 aeration tank of a municipal wastewater treatment plant (wwtp) in Groesbeek, The Netherlands

$141 \quad\left(51^{\circ} 45^{\prime} 34.3^{\prime \prime} \mathrm{N} 5^{\circ} 57^{\prime} 15.9^{\prime \prime} \mathrm{E}\right)$.

142 Unless stated otherwise, biomass was harvested from the bioreactors and wwtp by gentle centrifugation

143 (600 $\times g$ for $10 \mathrm{~min}$ ), washed twice, and subsequently resuspended in mineral salts medium to a final

144 density corresponding to approximately $100 \mu \mathrm{g}$ protein $\mathrm{ml}^{-1}$.

146 Inactivation of comammox Nitrospira by 1,700

147 To test for the irreversible inactivation of comammox Nitrospira by 1,7OD, biomass from the Ca. N. kreftii

148 enrichment culture (60) was harvested and resuspended in $50 \mathrm{ml}$ mineral salts medium without nitrogen

149 source containing $100 \mu \mathrm{M}$ 1,70D (99\% purity, Merck KGaA, Darmstadt, Germany) from a stock solution

150 (75.4 $\mathrm{mM})$ in dimethyl sulfoxide (DMSO). Cells were incubated for 10 minutes in the dark in a shaking

151 incubator $\left(150 \mathrm{rpm}, 24^{\circ} \mathrm{C}\right)$. Subsequently, incubations were supplemented with ammonium to a final

152 concentration of $350 \mu \mathrm{M}$ and were incubated for 72 hours. Control incubations receiving only $66.4 \mu \mathrm{l}$

153 DMSO without 1,7OD were performed in parallel. As a positive control for the inhibition of the ammonia 
154 oxidation activity, cells were incubated in the presence of $100 \mu \mathrm{M}$ allylthiourea (ATU; $\geq 98 \%$ purity, Merck

$155 \mathrm{KGaA}$, Darmstadt, Germany), which has been shown to inhibit comammox Nitrospira previously (2).

156 Finally, the specificity of $1,70 D$ as inhibitor of the ammonia-oxidizing activity was verified by incubating

157 comammox Nitrospira cells in the presence of $100 \mu \mathrm{M}$ 1,7OD and $20 \mu \mathrm{M}$ nitrite. All incubations were

158 performed in three biological replicates. During incubation, samples for the determination of ammonium,

159 nitrite and nitrate concentrations were taken every two hours for the first 8 hours of incubation, as well

160 as after 24,48 and 72 hours of incubation.

Analytical methods

163 Ammonium concentrations were measured fluorometrically using a modified orthophatal-dialdehyde 164 assay (61). Nitrite and nitrate concentrations were determined colorimetrically with the Griess reaction 165 (62) as described elsewhere (63). For the determination of total protein content, cells were lysed using 166 the Bacterial Protein Extraction Reagent (B-PER ${ }^{\mathrm{TM}}$, Thermo Fisher Scientific, Waltham, MA, USA) according

167 to manufacturer's instructions in combination with mild sonication (1 min, $20 \mathrm{~Hz}$ ). Post lysis, protein 168 concentrations were measured using the Pierce bicinchoninic acid protein assay kit (Thermo Fisher 169 Scientific, Waltham, MA, USA) according to the 'Enhanced Test-tube' protocol. All fluoro- and colorimetric 170 measurements were performed using a Tecan Spark ${ }^{\circledR}$ M10 plate reader (Tecan Trading AG, Männedorf, 171 Switzerland).

172

173 In situ ABPP-based fluorescent labelling of $A M O$ and $M M O$

174 Active biomass was harvested as described above, resuspended in $50 \mathrm{ml}$ mineral salts medium and 175 incubated with $100 \mu \mathrm{M}$ 1,70D for 30 minutes (150 rpm) in the dark. Subsequently, cells were pelleted by 176 gentle centrifugation (600 $\times g$ for 10 minutes), washed twice in sterile PBS, pH 7.5 and fixed using a 50\% $177(\mathrm{v} / \mathrm{v})$ ethanol/PBS solution for 10 minutes at room temperature (RT). Fixed biomass was washed once with 178 PBS and subjected to the CuAAC reaction, which was performed in plastic microcentrifuge tubes $(1,5 \mathrm{ml})$ 179 in a final volume of $250 \mu \mathrm{l}$. Biomass was resuspended in $221 \mu \mathrm{l}$ sterile PBS, mixed with $12.5 \mu \mathrm{l}$ of a 100 $180 \mathrm{mM}$ freshly prepared sodium ascorbate solution ( $\geq 99 \%$ purity, Merck KGaA, Darmstadt, Germany) and $181 \quad 12.5 \mu$ l of a $100 \mathrm{mM}$ freshly prepared aminoguanidine hydrochloride solution ( $\geq 98 \%$ purity, Merck KGaA). 182 A dye mixture containing $1.25 \mu \mathrm{l}$ of a $20 \mathrm{mM} \mathrm{CuSO}_{4}$ solution (99,99\% purity, Merck KGaA), $1.25 \mu \mathrm{l}$ of a 100 $183 \mathrm{mM}$ Tris(3-hydroxypropyltriazolylmethyl)amine solution (THPTA; 95\% purity, Merck KGaA) and $0.3 \mu \mathrm{l}$ of 5 $184 \mathrm{mM}$ Azide-Fluor 488 in DMSO ( $\geq 90 \%$ purity, Merck KGaA) was incubated in the dark for 3 minutes. 185 Subsequently, the dye mixture $(2.8 \mu \mathrm{l})$ was added to the CuAAC reaction tubes. The tubes were gently 
mixed and incubated for 60 minutes (RT, in the dark). CuAAC reactions were terminated by harvesting the cells by gentle centrifugation ( $600 \times g$ for 10 minutes). Cell pellets were washed three times with sterile PBS to remove unbound fluorophores and either used directly for downstream analyses, or resuspended in a $50 \%(\mathrm{v} / \mathrm{v})$ ethanol/PBS solution and stored at $-20^{\circ} \mathrm{C}$.

\section{Fluorescence in situ hybridization and microscopy}

192 AMO/MMO labelled biomass was hybridized with fluorescently labelled oligonucleotides as described by 193 Daims et al. (64). Probes used in this study (Table S1) were 5' or 5' and 3'-labelled with the dyes Cy3 or

194 Cy5 (65). After hybridization and washing, slides were dried and embedded in DAPI-containing 195 Vectashield ${ }^{\circledR}$ antifading mounting medium (Vector Laboratories Inc., Burlingame, CA). Probe and ABPPconferred fluorescence was recorded using a Leica TCS Sp8x confocal laser microscope (CLSM; Leica Microsystems B.V., Amsterdam, the Netherlands) equipped with a $405 \mathrm{~nm}$ UV diode and a pulsed white 198 light laser. Images were recorded using $63 \times$ or $100 \times$ oil immersion objectives at a resolution of $1991,024 \times 1,024$ pixels and 8-bit depth.

200 The spatial distribution of the ABPP-based AMO/MMO fluorescent signal within bacterial cells was 201 investigated using the HyVolution deconvolution module of the Huygens Essential Suite (Scientific Volume 202 Imaging B.V, Hilversum, The Netherlands). N. europaea, N. inopinata, M. oryzae and M. tundarae were 203 used as representatives of canonical $A O B$, comammox bacteria, and pMMO and sMMO-only containing 204 MOB, respectively. Images were acquired using a Leica Sp8x CLSM with a 100x oil immersion objective, a $2050.5 \mathrm{AU}$ pinhole size at a resolution of $1,024 \times 1,024$ pixels, and were deconvolved using the resolutionoptimized algorithm of the HyVolution module.

Immunogold localization of the AMO and PMMO enzymes and quantification

209 Active cells of $N$. europaea and M. oryzae were inactivated using 1,70D as described above, harvested, 210 fixed for 30 minutes at RT (2\% PFA, 0.5\% GA in $0.1 \mathrm{M}$ phosphate buffer $\mathrm{pH}$ ) and processed for Tokuyasu 211 sectioning $(66,67)$. Cells that were incubated in the presence of DMSO without 1,70D were used as a 212 control. After trimming in order to provide a suitable block face, $65 \mathrm{~nm}$ sections were cut on a $35^{\circ}$ diamond 213 knife (Diatome, Biel/Bienne, Switzerland) at $-100^{\circ} \mathrm{C}$ in a cryo-ultramicrotome (UC7/FC7 Leica 214 Microsystems, Vienna, Austria), picked up using a mixture of $1 \%$ methylcellulose in $1.2 \mathrm{M}$ sucrose (68) and 215 transferred to 100\#H copper grids (Agar Scientific LTD., Essex, UK).

216 In order to successfully localize the AMO/pMMO enzymes in the bacterial cells, the thawed cryo-sections 217 were subjected to a CuAAC reaction (as described above) using $5 \mathrm{mM}$ biotin-azide in DMSO (>95\% purity, 
218 Jena Bioscience $\mathrm{GmbH}$, Jena, Germany) instead of Azide-Fluor 488. Subsequently, immuno-gold

219 localization of the biotinylated enzymes was performed according to Slot and Geuze (2007) using a rabbit

220 anti-biotin antibody (ab53494, Abcam plc., Cambridge, UK, diluted 1:600 in 1\% BSA-C) and 10 nm protein-

221 A gold (CMC-UMC Utrecht, the Netherlands). Sections were embedded in methyl-cellulose containing

$2220.2 \%$ uranyl-acetate before imaging using a JEOL JEM-1400 flash transmission electron microscope,

223 operating at $120 \mathrm{KV}$.

224 The localization of the immuno-gold labels was counted in nine to twelve cells for each sample and was

225 classified in one out of four classes: i) resin, ii) membrane-associated, iii) cytoplasmic and iv) unclear. To

226 this end, circles with a diameter of $15 \mathrm{~nm}$ were drawn around the center of each gold particle. The gold

227 particles themselves had a given diameter of $10 \mathrm{~nm}$ and the antibody-protein A complex accounted for

228 another $10 \mathrm{~nm}$, thus the epitopes the antibodies were bound to were expected to lie somewhere within

229 these $15 \mathrm{~nm}$ circles. In case gold particles were located in the cytoplasm but with a membrane present

230 within the circle, the label localization was classified as unclear.

231 The location of the epitopes that classified as 'membrane-associated' was investigated in greater detail.

232 More specifically, labels on the periplasmic side of the intracytoplasmic membrane compartments were

233 classified as 'periplasmic' while labels present on the cytoplasmic side were classified as 'cytoplasmic'.

234 Labels localized on transversely sectioned membrane stacks or exactly on top of membranes, were

235 classified as unclear.

237 Effect of growth stage on ABPP-based fluorescent labelling efficiency

238 Cells of $N$. europaea were grown as described above in buffered medium (4.8 g/L HEPES). Control

239 incubations with $N$. europaea cells grown in the absence of ammonium as well as abiotic controls were

240 performed in parallel. All incubations were performed in two biological replicates. Samples $(10 \mathrm{~mL})$ were

241 taken regularly to monitor protein content, ammonium concentration and $\mathrm{pH}$. Additional samples (50 $\mathrm{mL})$

242 were subjected to the ABPP-based labelling protocol as described above. For the quantification of the

243 relative biovolume fractions that depicted an AMO labelling, as well as the intensity of the signal, the

244 ABPP-based protocol was combined with FISH using an equimolar mixture of the probes NSO190,

245 NSO1225 and NEU653 (Cy3) and EUB338mix (Cy5) (Table S1). Subsequently, 15 image pairs were recorded

246 per sample at random fields of view using the Leica TCS Sp8x CLSM. The images were imported into the

247 image analysis software daime (69) and evaluated as described elsewhere (70). 
Biomass from a nitrifying enrichment culture and activated sludge from a municipal wwtp were subjected to the ABPP-based AMO/MMO labelling protocol. For both biomasses, three types of control incubations

252 were performed in parallel. These contained (i) 1,70D without the addition of the fluorescent azide253 labelled dye in the CuAAC reaction, (ii) DMSO without 1,70D during the initial incubation, with complete

254 subsequent CuAAC reaction, and (iii) DMSO without 1,7OD during the initial incubation and without 255 addition of the fluorescent azide-labelled dye in the CuAAC reaction. After labelling, biomass was 256 dispersed by mild sonification $\left(30 \mathrm{~s}\right.$ at $20 \mathrm{~Hz}$ ) and stored at $-80^{\circ} \mathrm{C}$ in glycerol-TE (GlyTE) buffer ( $10 \mathrm{mM}$ Tris$257 \mathrm{HCl}, 1 \mathrm{mM}$ EDTA pH 8.0, 5\% v/v glycerol) until further processing. Samples were thawed on ice and 258 subjected to fluorescence-activated cell sorting (BD FACSMelody ${ }^{\mathrm{TM}}$, BD Biosciences, NJ, USA). Based on 259 the initial cell density determined by FACS, samples were diluted up to 1:4 with sterile PBS to ensure a 260 final event rate of $<10.000$ events $\sec ^{-1}$. Control incubations were used to determine a gating strategy for 261 the specific sorting of the labelled population in the samples that best excluded unlabeled and 262 autofluorescent cells. Subsequently, DNA was extracted from the bulk-sorted cell clusters, the unsorted 263 control incubations and the initial biomass, followed by metagenomic sequencing.

264 Additionally, we tested for potential biases on the community composition caused by treatment steps 265 unrelated to the ABPP-based protocol. These included potential effects of washing steps, biomass fixation, 266 sonication and storing conditions. For this purpose, biomass from the nitrifying enrichment culture was 267 subjected to different combinations of physical and chemical treatments occurring during the ABPP-based 268 protocol (Table S2), followed by DNA isolation and metagenomic sequencing (see below).

DNA extraction and metagenomic sequencing

271 Total DNA from all samples was extracted using the DNeasy Blood \& Tissue Kit (Qiagen Ltd., West Sussex,

272 UK) according to manufacturer's instructions. Genomic sequencing libraries were prepared using the 273 Nextera XT Kit (Illumina, San Diego, CA, USA) following manufacturer's recommendations using a total of $2741 \mu \mathrm{g}$ of input DNA, normalized to a concentration of $0,2 \mathrm{ng} / \mu \mathrm{l}$. The libraries were sequenced using an 275 Illumina MiSeq with MiSeq Reagent Kit v.3 (2x 300 bp, Illumina, San Diego, CA, USA) according to 276 manufacturer's instructions.

\section{Metagenome assembly and binning}

279 Quality-trimming, adapter removal and contaminant filtering of Illumina paired-end sequencing reads was 280 performed using BBDuk (BBTools version 37.76; with settings $k t r i m=r, k=23$, mink=11, hdist=1, qtrim=rl, 281 trimq=17, $\operatorname{maq}=20$, maxns=0, minlen=150) (71). Processed reads from all libraries from the same 
biological sample were de novo co-assembled using metaSPAdes v3.11.1 (72) with default settings, which

283 iteratively assembled the metagenome using $k$-mer sizes of $21,33,55,77,99$ and $127 \mathrm{bp}$. After assembly,

284 reads were mapped back to the metagenomic contigs for each sample separately using Burrows-Wheeler

285 Aligner (BWA version 0.7.17) (73), employing the "mem" algorithm. The mapping files were processed

286 and converted using SAMtools version 1.9 (74). Metagenomic binning was performed including all contigs

$287 \geq 1,500 \mathrm{bp}$, using differential coverage, sequence composition and linkage information. To optimize the

288 binning results, five different binning algorithms were used: BinSanity v0.2.6.1 (75), COCACOLA (76),

289 CONCOCT (77), MaxBin 2.0 v2.2.4 (78) and MetaBAT 2 v2.12.1 (79). The five bin sets were supplied to DAS

290 Tool v1.0 (80) for consensus binning, yielding the final optimized bins. Bin quality and completeness were

291 assessed based on single-copy marker gene presence by CheckM v1.0.7 (81). Phylogenetic assignment of

292 the final bins was predicted using the classification workflow of GTDB-Tk v0.2.1 (82).

293 Besides the assembling and binning method described above, the sequencing data retrieved from the

294 FACS-sorted activated sludge sample were assembled separately, with subsequent binning of all contigs

$295 \geq 1,500 \mathrm{bp}$ and manual refinement using the anvi'o metagenomics workflow (83). Here, contigs not

296 clustering with the rest of the bin, based on sequence composition or coverage, were inspected

297 individually in the assembly graph using Bandage v0.8.1 (84). Additionally, genes present on these contigs

298 were compared against the NCBI non-redundant nucleotide database using BLASTx (85). Contigs without

299 connections in the assembly graph and containing genes not matching the taxonomic affiliation of the bin

300 were removed from the respective bin. In order to achieve a consistent naming, these bins were linked

301 back to the ones obtained by the automated binning approach described above using dRep (v2.0.0.) with

302 a $99 \%$ identity cutoff (86).

303 For all samples, the total number of sequenced base pairs (bp) and reads, the number of aligned bp per

304 contig, and the number of mapped reads per contig was obtained as described above. Final bins were

305 annotated using PROKKA v1.12-beta (87) against the RefSeq bacterial non-redundant protein database

306 (release 92).

\section{Marker gene recovery and read recruitment}

309 Genes on assembled contigs $\geq 1,500$ bp were identified by Prodigal (88). The translated amino acid

310 sequences were mined using Hidden Markov Models (HMM) available from the Pfam database to extract

311 the assembled genes for ammonia monooxygenase subunit A (amoA, PF02461) and RNA polymerase beta

312 subunit $(r p o B, P F 04563)(89,90)$. Genes with a sequence score less than 20 or an e-value greater than $1 \mathrm{e}-$

3135 were inspected manually to remove false-positives. Recovered amino acid sequences were queried 
314 (blastp version 2.10.0+ -evalue 1e-50, -culling_limit 10, -qcov_hsp_perc 90,-max_hsps 5) against the NCBI

315 non-redundant protein database (version 5) to infer their taxonomic affiliations, and to identify gene

316 fragments (less than $45 \%$ length of closest reference), which were excluded from quantitative analyses

317 (91). Nucleotide sequences of the genes and assembled contigs $\geq 1,500$ bp were used to quantify recruited

318 reads using BBMap (BBTools version 37.76 idfilter=0.99, pairedonly=t, ambiguous=random) (71). Read

319 recruitment per gene and sample was normalized by gene length and sequencing depth as paired Reads

320 per Kilobase gene length per Million reads recruited to the assembly (RPKM). R was used to normalize

321 read abundances, calculate correlations, and for visualization $(92,93)$.

\section{Data availability}

324 Sequencing data obtained in this study have been deposited in the National Center for Biotechnology

325 Information (NCBI) database under BioProject accession numbers PRJNA691748 (nitrifying enrichment

326 culture) and PRJNA691751 (activated sludge sample).

328 Results

329 Inhibition of ammonia oxidation in comammox by 1,70D

330 The ability of 1,70D to act as an irreversible and mechanism-based inactivator of the AMO enzyme in the 331 canonical AOB Nitrosomonas europaea was previously demonstrated (19), and the pMMO of 332 Methylococcus capsulatus (Bath) was shown to be partially inhibited by alkynes with chain lengths $\leq \mathrm{C}_{7}$ 333 (94). However, comammox Nitrospira harbor a phylogenetically distinct AMO enzyme $(1,2,36)$. In order 334 to test the efficiency of inactivation of the comammox AMO by 1,7OD, inhibition of ammonia oxidation

335 activity was determined in the $\mathrm{Ca}$. N. kreftii enrichment culture, which contained comammox Nitrospira 336 as the sole ammonia-oxidizing microorganism (60).

337 In the absence of $1,70 D$, the culture oxidized $350 \mu \mathrm{M}$ ammonium in 48 hours to nitrate without 338 intermediate nitrite accumulation. In contrast, addition of $100 \mu \mathrm{M} 1,70 \mathrm{D}$ resulted in a rapid and complete 339 inhibition of the ammonia-oxidizing activity, which was comparable to the inhibitory effect of $100 \mu \mathrm{M}$ ATU

340 (Figure 1A, B). No influence of 1,70D or ATU on the nitrite-oxidizing activity of the culture was detected 341 (Figure 1C, D).

343 In situ fluorescent labelling of $A M O$ and $M M O$

344 We adapted the ABPP-based AMO labelling protocol developed by Benett et al. (19) to allow the in situ 345 fluorescent labelling of ammonia and methane-oxidizing bacteria. Biomass containing different ammonia 
or methane oxidizers was incubated in the presence of $100 \mu \mathrm{M}$ 1,70D, which lead to the formation of stable 1,7OD-enzyme complexes within the bacterial cells $(19,47)$. After EtOH fixation, the inactivated biomass was subjected to a highly specific CUAAC reaction. The use of the CUAAC reaction enabled the covalent coupling of the alkyne-labelled enzymes to an azide-labelled Fluor488 dye, which resulted in the fluorescent labelling of AMO (Figure 2) and MMO-containing microorganisms (Figure 3). The efficiency and specificity of the ABPP-based AMO labelling was verified using pure cultures of the following nitrifying microorganisms: (i) Nitrosomonas europaea, a well-studied canonical ammoniaoxidizing bacterium, (ii) Nitrospira inopinata, the only comammox bacterium available as pure culture (58), (iii) Nitrospira moscoviensis, a canonical nitrite-oxidizing bacterium and (iv) Nitrosocosmicus franklandus, an ammonia-oxidizing archaeon. Efficient staining of the N. europaea and N. inopinata cells was observed when 1,70D-treated cells were subjected to the CuAAC reaction (Figure 2A, C). In contrast, no fluorescent signal was obtained when the same cells were not treated with 1,7OD (Figure 2B, D), or in the canonical, nitrite-oxidizing $N$. moscoviensis (Figure 2F). However, also $N$. franklandus cells did not show any fluorescent labelling (Figure 2E), indicating that the ABPP-based protocol was not able to label the archaeal AMO enzyme.

Although it has been reported that MMOs and AMOs exhibit similar inhibition patterns (35) and it was shown that both sMMO and PMMO enzymes can also be inactivated by acetylene as well as other alkyne compounds $(47,51,52)$. The effect of $1,70 D$ on methane-oxidizing activity has not been studied yet. To test whether the 1,70D-based ABPP protocol could also label MMOs the following pure cultures were subjected to our labelling protocol: (i) Methylotetracoccus oryzae, a type lb methanotrophic gammaproteobacterium (56); (ii) Methylosinus sporium, a type II methanotrophic alphaproteobacterium (54); (iii) Methylacidiphilum fumariolicum, an acidophilic methane oxidizer of the phylum Verrucomicrobia (6); and (iv) Methylocella tundrae, an alphaproteobacterial methanotroph encoding only sMMO (55). Specific 1,7OD-dependent fluorescent staining of all tested methanotrophic cultures indicated efficient ABPP-based labelling of MOB, regardless of enzyme type and phylogenetic affiliation (Figure 3 ).

372 Correlation of the AMO/MMO staining with the bacterial growth stage

373 Laboratory cultivation conditions poorly reflect the conditions microorganisms encounter in natural and 374 engineered ecosystems. In these environments, fluctuations in substrate and nutrient availability rarely 375 permit the continuous growth of microorganisms, including AOB and MOB (95). To investigate the 376 potential effect of the bacterial growth stage on the efficiency of the AMO-based staining, a N. europaea 377 pure culture was cultivated in the presence of $10 \mathrm{mM}$ ammonium and ammonia-oxidizing activity and 
growth were monitored over 21 days. The culture stoichiometrically oxidized ammonium to nitrite (Figure $4 \mathrm{~A})$ and biomass samples taken at different time points were subjected to the ABPP-based protocol. The AMO-derived fluorescent signal intensity reached its maximum at the start of the exponential growth phase (day 7) and decreased when the culture entered early stationary phase, but maintained a low signal intensity (35\% compared to the maximum intensity) even in late stationary phase (Figure 4B). Throughout all growth stages of the culture a high percentage (>90\%) of cells was stained, with a small decrease (16\%) in staining efficiency observed only in late stationary phase (Figure 4B).

Subcellular localization of active AMO and MMO enzymes

AMO and pMMO are known to be membrane-integral enzymes, with their active center on the periplasmic face of the cytoplasmic membrane or the intracytoplasmic membrane (ICM) systems $(96,97)$. In contrast, the SMMO is a soluble cytoplasmic enzyme (37). To confirm these subcellular localizations, pure cultures of the canonical ammonia oxidizer N. europaea, the comammox bacterium N. inopinata, the type Ib methanotroph $M$. oryzae (containing only $\mathrm{pMMO}$ ), and the methanotroph $M$. tundrae (containing only sMMO) were subjected to the ABPP-based AMO/MMO-staining protocol in combination with FISH and subsequent deconvolution fluorescence microscopy (Figure 5). In N. europaea the AMO label was observed mainly in a thick layer surrounding the cytoplasm (Figure 5A), coinciding with the localization of the cytoplasmic membrane and the peripheral organization of the ICM system in Nitrosomonas (97-99). In M. oryzae the ICMs are arranged as stacks of vesicular discs located in the central cytoplasm (96), which agrees with the observed strong pMMO fluorescent staining on apparently organized structures within the cytoplasm (Figure 5B). As expected, in M. tundrae the sMMO labelling overlapped with the cytoplasmic 16S rRNA-derived signal (Figure 5C). Unfortunately, the small cell size ( $\leq 1 \mu \mathrm{m}$ ) of $N$. inopinata precluded an unambiguous localization of the AMO-derived fluorescent signal (data not shown). In order to examine the localization of active AMO and pMMO enzymes in more detail, cells of $N$. europaea and $M$. oryzae were subjected to a modified ABPP-based AMO/MMO-staining protocol. In this experiment, the CUAAC reaction was used to biotinylate the $\mathrm{AMO} / \mathrm{pMMO}$ enzymes within active bacterial cells, with subsequent immunogold labelling and transmission electron microscopy, thus providing highresolution information on the subcellular localization of the complexes (Figure 6). In N. europaea, 74\% of the immunogold labelling indicated a membrane localization of the AMO complex, and the majority of 407 these gold particles (49\%) appeared to face the periplasmic space, with only $13 \%$ oriented towards the 408 cytoplasm (Figure 6B-C, Table S3). In accordance to the proposed localization of the alkynylation site in 409 the MOB Methylococcus capsulatus (47), 61\% of gold labelling M. oryzae depicted a membrane 
410 localization, with $71 \%$ of these membrane-associated labels on the periplasmic side of the membrane

411 (Figure 6E-F, Table S3). In both cases gold particles were seldomly found in the cytoplasm or outside the

412 cells, indicating the high specificity of the labelling protocol (Figure 6, Table S3).

414 In situ ABPP-based fluorescent labelling of ammonia oxidizers in complex communities

415 The ability of the ABPP-based AMO/MMO staining protocol to label ammonia oxidizers present in complex

416 microbial communities was assessed in nitrifying enrichment cultures and activated sludge samples. First,

417 we tested our newly developed protocol on an enrichment culture of $\mathrm{Ca}$. N. nitrosa and $\mathrm{Ca}$. N. nitrificans

418 (2) and a nitrifying co-culture containing canonical AOB, comammox and canonical Nitrospira.

419 Furthermore, we demonstrated that combination of the ABPP-based protocol with 16S rRNA-targeted

420 FISH allowed the simultaneous phylogenetic identification of the ammonia-oxidizing bacterial populations

421 in all samples (Figure 7).

422 The ABPP-based protocol was able to specifically and efficiently stain both types of ammonia-oxidizing

423 bacteria present in these mixed communities. AMO-derived fluorescent labelling of comammox bacteria

424 was observed both in the enrichment culture containing $\mathrm{Ca}$. N. nitrosa and $\mathrm{Ca}$. N. nitrificans (Figure 7A,

425 B), and in the nitrifying enrichment (Figure 7C, D). In the latter, betaproteobacterial canonical AOB were

426 also efficiently double-stained by the ABPP-based protocol and FISH, while some Nitrospira were detected

427 by FISH only, presumably corresponding to canonical Nitrospira present in this sample.

Targeted metagenomics

430 In combination with fluorescence-activated cell sorting, our ABPP-based staining protocol could be 431 employed for targeted metagenomics of $\mathrm{AMO}$ and $\mathrm{MMO}$-containing bacteria present in complex 432 environmental samples. To prove the feasibility of this approach, we tested the protocol on two samples 433 of different complexity, a nitrifying enrichment culture and activated sludge from a full-scale wastewater 434 treatment plant.

435 Successive to the application of the ABPP protocol and floc disruption, biomass of the nitrifying 436 enrichment cultures was used for fluorescence-activated cell sorting, with pooled collection of all positive 437 sorting events (Figure S1). This fraction was subjected to DNA extraction and metagenomic sequencing, 438 along with an untreated biomass sample and a range of controls to test for potential biases in the protocol 439 (Table S2). Subsequent metagenomic consensus binning allowed the recovery of three high quality ( $>92 \%$ 440 completeness and $<3.7 \%$ redundancy) metagenome assembled genomes (MAGs) of ammonia-oxidizing 441 microorganisms from the nitrifying enrichment, corresponding to one Nitrosomonas (maxbin2.003) and 
442 two Nitrospira species (metabat2.63_sub, metabat2.21) that were strongly enriched in the metagenome

443 of the sorted sample (Figure S2, Table S4). Of the two recovered Nitrospira MAGs, only metabat2.21

444 contained an amoA sequence, whereas this gene was absent in MAG metabat2.63_sub. However, an

445 unbinned contig containing an amoA sequence showed similar coverage to this MAG across all samples,

446 suggesting its association with this Nitrospira MAG. All MAGs corresponding to non-ammonia-oxidizing

447 microorganisms were de-enriched in the targeted metagenome (Figure S2, Table S4), indicating a

448 successful targeted retrieval of AMO-containing bacteria.

449 In order to demonstrate the efficacy of the labelling and sorting protocol in a manner unbiased by

450 metagenomic binning approaches and different sequencing depths, we used metagenomic read mapping

451 to assembled and extracted $a m o A$ as functional marker for ammonia-oxidizing microorganisms, and $r p o B$,

452 a conserved single-copy phylogenetic marker gene to detect and differentiate all members of the

453 microbial community. In total, ten genes belonging to the copper-containing membrane-bound

454 monooxygenase family (Pfam PF02461) were identified in the co-assembled contigs retrieved from the

455 nitrifying enrichment: three were highly similar to amoA of Nitrosomonas and two of comammox

456 Nitrospira (>96\% amino acid identity), while the remaining five were most similar to unresolved genes

457 within proteobacterial lineages not known to oxidize ammonia. These latter genes belonged to a group of

458 putative hydrocarbon monooxygenases $(\mathrm{HMO})(100,101)$ and became undetectable in the sorted sample,

459 and thus were excluded from further analysis. A total of 48 rpoB genes were recovered and analyzed,

460 including two affiliated with Nitrosomonas (>90\% amino acid identity), two with comammox Nitrospira

461 (>97\% amino acid identity), and six with canonical Nitrospira (>97\% amino acid identity). For both marker

462 genes belonging to the dominant ammonia oxidizers in the bioreactor, the normalized read abundances

463 increased in the sorted samples compared to the unsorted samples - Nitrosomonas and comammox

464 Nitrospira amoA increased 41- and 13-fold, and their rpoB 86- and 12-fold, respectively (Figure 8). This

465 increase in abundance was decoupled from the total number of reads per sample recruited to the co-

466 assembly, indicating the changes were not due to variations in sequencing depth (Figure 8). Finally, the

467 abundance of non-ammonia-oxidizing community members' rpoB genes decreased from $93.6 \%$ of the

468 total normalized read abundance to $12.5 \%$, indicating a strong de-enrichment of the microbial community

469 members lacking AMO (Figures 8 and S2). Therefore, the ABPP-based fluorescent labeling coupled to cell

470 sorting successfully and specifically enriched the ammonia-oxidizing microorganisms present in the

471 nitrifying bioreactor.

472 However, the ABPP-based labelling and sorting approach did reveal biases and limitations. First, there

473 appeared to be enrichment of nitrifying bacteria due to treatment steps prior to sorting, particularly 
474 among the sonicated and ethanol-treated samples, but also by the CuACC reaction itself (Figure 8). These

475 effects may be due to their tendency to grow as microcolonies within biomass aggregates, which may 476 protect them from the effects of sonication, ethanol fixation, and the CuAAC reaction (92-94). Second,

477 the apparent most abundant ammonia-oxidizer after ABPP-based labelling and cell sorting depended on

478 the marker gene, as a Nitrosomonas-like canonical AOB appeared to be most highly enriched based on

$479 a m o A$ abundance increases, while this was a comammox Nitrospira in the $r p o B$ analysis. The slopes of

480 linear correlations $\left(r^{2}>0.95\right.$, Figure S3) of these most abundant $a m o A$ and $r p o B$ genes support genomic

481 evidence that this discrepancy may be an effect of the copy number of amoA, i.e. Nitrosomonas typically

482 encode three copies of amoA but comammox Nitrospira only one (102). Third, the larger increases in

483 abundance of both Nitrosomonas marker genes compared to comammox Nitrospira suggest that the

484 technique may slightly favor Nitrosomonas-like $A O B$. Fourth, some amoA and rpoB affiliated with $A O B$

485 were either poorly enriched or even de-enriched, which makes it tempting to speculate on lifestyles

486 independent of ammonia oxidation that might result in absence of the AMO enzyme in these cells (Figure

487 8). While important to acknowledge and account for these caveats, metagenomic recovery and

488 abundance estimations clearly showed effective enrichment of ammonia-oxidizing microorganisms by the

489 ABPP-labelling protocol coupled to fluorescence-activated cell sorting.

490 When applied to activated sludge sampled at a municipal wwtp, the ABPP-based protocol in combination

491 with cell sorting (Figure S1) and metagenomic sequencing facilitated a 128-fold increase in coverage of

492 the most abundant Nitrosomonas species MAG (metabat2.58) in comparison to the untreated sample,

493 demonstrating its successful and targeted enrichment (Figure 9, Table S5). In general, canonical ammonia

494 oxidizers were of extremely low abundance in this wwtp, and this MAG only contributed $0.14 \%$ of all

495 mapped reads in the metagenome of the untreated sample. After cell sorting and sequencing, it was

496 enriched to account for $8.64 \%$ of the mapped reads, which allowed the recovery of a high-quality draft

497 genome with estimate completeness of $100 \%$ and $0.81 \%$ redundancy (Figure 9 , Table S5). A second MAG

498 (metabat2.38) affiliated with the genus Nitrosomonas accounted for $0.12 \%$ and $0.96 \%$ of mapped all reads

499 in the samples before and after sorting respectively, corresponding to an 8-fold increase in coverage in

500 the sorted sample (Table S5). This clearly demonstrates the efficacy of our ABPP-based protocol for the

501 targeted recovery of ammonia-oxidizing bacteria, even when they constitute a minute fraction of the

502 original community.

503 Surprisingly, a high percentage (65.2\%) of the total reads obtained for the sorted activated sludge sample

504 were affiliated with putative glycogen-accumulating organisms (GAOs) of the family Competibacteraceae

505 (Tables S5). We obtained in total 7 MAGs (>70\% completeness) affiliated with this family, which showed 
an increase in coverage from 8.9 to 52 -fold in the sorted compared to the untreated metagenomic sample

507 (Figure 9, Table S5). However, none of these MAGs contained genes encoding AMO or MMO subunits, 508 although some unbinned amoA sequences were present in the full assembly. Thus, we performed manual 509 binning and bin refinement using a new assembly of the sequencing reads of the sorted sample only, but 510 using differential coverage information from all samples. These refined MAGs were then screened for 511 genes involved in ammonia or methane oxidation, or genes which otherwise might explain the enrichment 512 of these GAOs by the ABPP-based sorting method. The manual binning allowed us to link the observed 513 amo genes to the dominant Nitrosomonas-affiliated MAG, but revealed no traces of an ammonia-oxidizing 514 potential for any of the Competibacteraceae family members (three Contendobacter and one 515 Competibacter species with an estimated genome completeness $>70 \%$; Table S6), and also MMO genes 516 were not detected in these MAGs. This indicates that the observed enrichment was not a result of an 517 AMO/MMO-based labelling and the only enzymes encoded by these Competibacteraceae MAGs known 518 to interact with alkynes and thus potentially explaining their fluorescent labelling (Figure S4) were 519 nitrogenases (103).

520 Consequently, to test for potential labelling of the nitrogenase complex, we incubated pure cultures of 521 the diazotroph Krypidia spormannii FAVT5 (59) and the methanotroph M. oryzae (56) under nitrogen522 fixing conditions. Subsequently, both cultures were subjected to the ABPP-based protocol. As M. oryzae 523 contains a pMMO, staining of these cells was observed, but compared to a culture grown in the presence 524 of nitrate as $\mathrm{N}$-source in the medium, the staining intensity was even slightly reduced (Figure S5). For $K$. 525 spormannii FAVT5 the amount of signal observed after the CuAAC reaction was just above background, 526 corresponding to approximately $11 \%$ and $25 \%$ of the signal obtained from active $N$. europaea and $M$. 527 tundrae cultures, respectively (Figure S5). Together, these results indicate that the presence of an active 528 nitrogenase does not contribute to the ABPP-based labelling, making the reason for the observed staining 529 of the Competibacteraceae-affiliated MAGs unclear.

\section{Discussion}

532 ABPP-based protocols have been successfully employed for the study of many microbial protein families, 533 such as proteases, kinases, hydrolases and glycosidases $(8,17)$. However to date, they have not been 534 applied for the labelling of whole cells within complex microbial communities, mainly due to the presence 535 of the microorganisms within a complex undefined matrix. This poses major challenges for employing the 536 CuAAC reaction, probe penetration into the cells, and labelling specificity and efficiency (104). The ABPP537 based protocol developed in this study, to our knowledge for the first time, overcomes these challenges 
and allows the fluorescent visualization of catalytically active enzymes in situ. The protocol makes use of the alkyne 1,70D, which serves as a bifunctional enzyme probe for the specific detection of AMO and MMO enzymes. The feasibility of this approach was previously demonstrated in vitro for the AMO of canonical $A O B$ (19), but had not been tested for labelling whole cells, phylogenetically distinct ammonia oxidizing microorganisms, or MMOs of methanotrophs. Generally, $n$-terminal and sub-terminal alkynes are known mechanism-based inactivators of both $\operatorname{AMO}(48,49)$ and MMO enzymes $(46,51)$. Here, we could show that $1,70 D$ inhibits ammonia oxidation in comammox Nitrospira (Figure 1) as efficiently as was reported for canonical $A O B$ (19). Furthermore, strong labelling of different $M O B$ harboring either the soluble or particulate MMO (Figure 3) indicated that also methanotrophs will be inhibited by this diyne. Application of the in situ ABPP-based protocol on pure and enrichment cultures of different canonical $\mathrm{AOB}$, comammox Nitrospira, and $\mathrm{MOB}$ resulted in their efficient and specific labelling (Figures 2 and 3 ), which directly conveyed information on the functional potential of the detected microorganisms in contrast to conventional in situ detection methods like FISH. Using high-resolution deconvolution light microscopy as well as electron microscopy, the ABPP-based protocol furthermore permitted visualization of the subcellular localization of the AMO and MMO enzymes (Figures 5 and 6). In accordance with previous studies $(55,98,99)$, the AMO and pMMO-derived fluorescent signals were mostly localized along the cytoplasmic membrane and on the intracytoplasmic membrane stacks in N. europaea and M. oryzae, respectively, whereas a cytoplasmic localization of the sMMO-derived signal was observed for $M$. tundrae. Furthermore, the immuno-gold labelling data obtained in this study suggested that the alkynylation sites for both the AMO and pMMO complexes were found on the periplasmic face of the ICMs. This congruence with the expected enzyme localizations further verified the suitability of the ABPP-based protocol for the specific labelling of AMO/MMO enzymes, and opens up possibilities for studying differences in enzyme distribution along intracellular structures in $A O B$ or $\mathrm{MOB}$ types with divergent cell morphologies. Simultaneously, the biotinylation of these enzymes via the ABPP-based protocol as tested here provides a new methodology for the profiling, affinity purification and study of AMO/MMO enzymes. In most natural and engineered systems microorganisms rarely encounter optimal growth conditions and

564 might be in very different growth stages. Previous studies have identified preservation of basal activity 565 levels of ammonia and methane oxidizers as a general adaptation mechanism used to cope with 566 alternating conditions and fluctuating substrate supply $(95,105)$. This indicates that they maintain a basal $567 \mathrm{AMO} / \mathrm{MMO}$ content throughout their life cycle. Quantification of ABPP-derived fluorescent signals in a $N$. 568 europaea pure culture showed a strong correlation between signal intensity and growth state (Figure 4). 569 As expected, the strongest signals were observed during the exponential growth phase, corresponding to 
570 a high AMO content of the cells. However, detectable ABPP-based labelling was observed even in the late 571 stationary phase of the culture, demonstrating that basal AMO contents are maintained also in non572 replicating cells, which however did not encounter substrate limitation. Thus, our ABPP-based protocol 573 apparently is suitable for the detection of ammonia and methane-oxidizing bacteria also under suboptimal 574 growth conditions.

575 As the ABPP-based protocol can be combined with FISH, it allows to directly link the functional potential 576 of a microorganism to its identity in situ, which potentially even can lead to the identification of novel ammonia or methane oxidizers. The need for simple detection methods not requiring a priori knowledge on the identity of microorganism was showcased by the recent discovery of complete nitrification within members of the genus Nitrospira $(1,2)$. While amoA-targeting PCR-based methods have successfully been employed to detect the presence and abundance of comammox bacteria in complex microbial communities or environmental samples (106), the distinction between canonical and comammox Nitrospira based on their phylogenetic affiliation is still problematic. Consequently, it also is not possible to reliably detect comammox Nitrospira in 16S rRNA-targeted FISH, which hampers research on their role in full-scale biotechnological systems like drinking and wastewater treatment plants. In combination with $\mathrm{FISH}$, the ABPP-based AMO/MMO staining method described here overcomes this limitation and enables an easy and reliable differentiation of ammonia and nitrite-oxidizing Nitrospira in complex microbial communities (Figure 7). Unfortunately, it was not possible to efficiently stain the archaeal AMO using 1,70D as bifunctional enzyme probe (Figure 2E). This is in accordance with the low sensitivity of AOA to longer-chain length $\left(>C_{5}\right)$ alkynes and their reversible inhibition by 1-octyne (49). Nevertheless, as AOA are sensitive to inactivation by n-alkynes with short carbon backbones $\left(\leq C_{5}\right)(19)$, a modification of the ABPP-based

592 protocol employing a smaller enzyme probe might permit also labelling of AOA. However, the fact that 593 diynes smaller than 1,5-hexadiyne are extremely reactive and difficult to obtain will make the applicability 594 of this method for the detection of AOA challenging.

595 Generally, most nitrifiers and methane oxidizers are notoriously difficult to cultivate due to their slow 596 growth rates and susceptibility to contamination. Besides classical cultivation attempts, the identification 597 of novel ammonia and methane oxidizers to date was achieved using metagenomic sequencing (1-6, 107598 110) and molecular techniques $(106,111,112)$. These contemporary cultivation-independent tools have 599 been invaluable for providing insights into the distribution and ecological importance of these functional 600 groups. Despite the fact that metagenomic sequencing has provided a wealth of information on these 601 microorganisms, it is often limited by the ecosystems' complexity and required resource investments, and 
602 can fail to recover sufficient data to reconstruct genomes of low-abundance organisms. Furthermore, the

603 lack of PCR primers able to amplify novel and possibly divergent sequences can hinder the detection of

604 new species with novel metabolic capabilities. Additionally, linking novel sequence types detected in

605 metagenomic or PCR-derived datasets to a specific phylotype often is a highly challenging and time-

606 consuming task. Thus, there is a pressing need for simple detection methods that do not requiring $a$ priori

607 knowledge on the identity of microorganism.

608 To overcome these limitations, we combined the newly developed ABPP-based AMO/MMO staining 609 protocol with cell sorting and subsequent metagenomic sequencing of the sorted subpopulation.

610 Application of this protocol on a nitrifying bioreactor enrichment culture substantially and specifically

611 enriched the multiple types of AOP present in the biomass (Figure 8). While the physical and chemical

612 treatments of the biomass during the ABPP-based protocol were observed to introduce biases resulting

613 in a slight unspecific enrichment of microorganisms like Nitrosomonas and Nitrospira, which possibly can

614 be explained by an increased cell stability due to their tendency to form microcolonies $(113,114)$, only

615 the final sorting step of the labelled bacteria resulted in the high and specific enrichment of these AOP

616 (Figure 8).

617 When applied on activated sludge from a full-scale municipal wwtp, the ABPP-based labelling protocol

618 with subsequent cell sorting resulted in the targeted recovery of a Nitrosomonas MAG (Figure 9). This

619 MAG was of very low abundance in the original sample, but the 128-fold increase in coverage achieved

620 by our staining and sorting approach allowed the reconstruction of a high quality genome, demonstrating

621 the power of this ABPP-based protocol.

622 Surprisingly, also several MAGs belonging to members of the Competibacteraceae family were retrieved

623 from the activated sludge sample. Contendobacter and Competibacter species are glycogen-accumulating

624 organisms, which frequently are encountered in enhanced biological phosphorus removal systems. In

625 these systems, they compete with phosphate-accumulating organisms for resources under the cyclic

626 anaerobic 'feast' - aerobic 'famine' regime (115) and thus reduce the phosphate removal efficiency of

627 these wastewater treatment systems. Their main metabolism includes synthesis and storage of glycogen

628 and polyhydroxyalkanoates, but they are also able of fermentation, denitrification and nitrogen fixation

629 (116). However, neither the annotation of the obtained MAGs nor existing literature supports the

630 involvement of these Competibacteraceae species in the nitrification process or methanotrophy. The

631 reason of their labelling and sorting (Figures S1 and S4) through the ABPP-based protocol thus is unclear

632 and requires future research. 
633 In conclusion, we present a novel ABPP-based protocol that enables the efficient in situ labelling of

$634 \mathrm{AMO} / \mathrm{MMO}-c o n t a i n i n g ~ b a c t e r i a$ and facilitates the targeted retrieval of enriched metagenomes. Although

635 optimization of the protocol for difficult sample types that exhibit high background fluorescence will be

636 necessary, the developed ABPP-based labelling protocol is a significant milestone for integrating

637 phylogenetic and physiological information that can be adapted and applied to complex microbial

638 communities. Coupling this novel method with more common techniques can help reveal the functional

639 differences in biomass aggregates of phylogenetically indistinguishable bacteria, as well as enable the

640 recovery of new genomic representatives critical for engineered processes that account for only a small

641 portion of the microbial community. Therefore, the future application of this ABPP protocol will contribute

642 greatly to an improved understanding of the microorganisms involved in important carbon and nitrogen

643 cycle processes within a variety of ecosystems.

\section{Acknowledgements}

646 The authors would like to thank C. Hogendoorn, M. Ghashghavi, M.A.R. Kox, C.J. Sedlacek and L. 647 Lehtovirta-Morley for the generous provision of biomass. and T. van Alen and H. Harhangi for technical 648 assistance. We are grateful to H. Koch, M. Wagner and H. Daims for helpful discussions. D.S. and M.S.M.J. 649 were supported by the European Research Council (ERC Advanced Grant Ecomom 339880), J.F., G.S., L.K., 650 M.A.H.J.v.K and S.L. by the Netherlands Organisation for Scientific Research (NWO; Gravitation Grant 651 SIAM 024.002.002, 016.Veni.192.062 and 016.Vidi.189.050).

\section{Author Contributions}

654 S.L. conceived the presented research. D.S., M.A.H.J.v.K., M.S.M.J. and S.L. planned research. S.L. and 655 M.A.H.J.v.K supervised the project. D.S., G.S., R.J.M., J.F. and L.K. executed experiments and analyzed 656 data. D.S., M.A.H.J.v.K. and S.L. wrote the paper. All authors discussed results and commented on the 657 manuscript. 


\section{References}

659 1. Daims H, Lebedeva EV, Pjevac P, Han P, Herbold C, Albertsen M, et al. Complete nitrification by 660 Nitrospira bacteria. Nature. 2015;528(7583):504-9.

661 2. van Kessel MA, Speth DR, Albertsen M, Nielsen PH, Op den Camp HJ, Kartal B, et al. Complete 662 nitrification by a single microorganism. Nature. 2015;528(7583):555-9.

663 3. Könneke M, Bernhard AE, de la Torre JR, Walker CB, Waterbury JB, Stahl DA. Isolation of an 664 autotrophic ammonia-oxidizing marine archaeon. Nature. 2005;437(7058):543-6.

665 4. Dunfield PF, Yuryev A, Senin P, Smirnova AV, Stott MB, Hou S, et al. Methane oxidation by an 666 extremely acidophilic bacterium of the phylum Verrucomicrobia. Nature. 2007;450(7171):879-82.

667 5. Ettwig KF, Butler MK, Le Paslier D, Pelletier E, Mangenot S, Kuypers MMM, et al. Nitrite-driven 668 anaerobic methane oxidation by oxygenic bacteria. Nature. 2010;464(7288):543-8.

6696 6ol A, Heijmans K, Harhangi HR, Tedesco D, Jetten MSM, Op den Camp HJM. Methanotrophy 670 below pH 1 by a new Verrucomicrobia species. Nature. 2007;450(7171):874-8.

671 7. in ' $t$ Zandt $M H$, de Jong AEE, Slomp CP, Jetten MSM. The hunt for the most-wanted 672 chemolithoautotrophic spookmicrobes. FEMS Microbiology Ecology. 2018;94(6):fiy064-fiy.

673 8. Sadler NC, Wright AT. Activity-based protein profiling of microbes. Current opinion in chemical 674 biology. 2015;24:139-44.

675 9. Speers AE, Cravatt BF. Profiling enzyme activities in vivo using click chemistry methods. Chemistry 676 \& biology. 2004;11(4):535-46.

677 10. Rostovtsev VV, Green LG, Fokin VV, Sharpless KB. A stepwise Huisgen cycloaddition process: 678 Copper(I)-catalyzed regioselective "ligation" of azides and terminal alkynes. Angewandte Chemie 679 International Edition. 2002;41(14):2596-9.

680 11. Tornøe CW, Christensen C, Meldal M. Peptidotriazoles on solid phase: [1,2,3]-triazoles by 681 regiospecific copper(I)-catalyzed 1,3-dipolar cycloadditions of terminal alkynes to azides. The Journal of 682 Organic Chemistry. 2002;67(9):3057-64.

683 12. Köhn M, Breinbauer R. The Staudinger ligation-A gift to chemical biology. Angewandte Chemie 684 International Edition. 2004;43(24):3106-16.

685 13. Greenbaum D, Baruch A, Hayrapetian L, Darula Z, Burlingame A, Medzihradszky KF, et al. Chemical 686 approaches for functionally probing the proteome. Molecular \& Cellular Proteomics. 2002;1(1):60-8.

687 14. Kocaoglu O, Calvo RA, Sham LT, Cozy LM, Lanning BR, Francis S, et al. Selective penicillin-binding 688 protein imaging probes reveal substructure in bacterial cell division. ACS chemical biology. 689 2012;7(10):1746-53.

690 15. Joyce JA, Baruch A, Chehade K, Meyer-Morse N, Giraudo E, Tsai F-Y, et al. Cathepsin cysteine 691 proteases are effectors of invasive growth and angiogenesis during multistage tumorigenesis. Cancer Cell. 692 2004;5(5):443-53.

693 16. Yestrepsky BD, Kretz CA, Xu Y, Holmes A, Sun H, Ginsburg D, et al. Development of tag-free 694 photoprobes for studies aimed at identifying the target of novel Group A Streptococcus antivirulence 695 agents. Bioorganic \& medicinal chemistry letters. 2014;24(6):1538-44.

696 17. Wright AT, Cravatt BF. Chemical proteomic probes for profiling cytochrome p450 activities and 697 drug interactions in vivo. Chemistry \& biology. 2007;14(9):1043-51. 
18. Saghatelian A, Jessani N, Joseph A, Humphrey M, Cravatt BF. Activity-based probes for the proteomic profiling of metalloproteases. Proceedings of the National Academy of Sciences of the United States of America. 2004;101(27):10000-5. 19. Bennett K, Sadler NC, Wright AT, Yeager C, Hyman MR. Activity-based protein profiling of ammonia monooxygenase in Nitrosomonas europaea. Applied and environmental microbiology. 2016;82(8):2270-9.

20. McTavish $\mathrm{H}$, Fuchs JA, Hooper AB. Sequence of the gene coding for ammonia monooxygenase in Nitrosomonas europaea. Journal of Bacteriology. 1993;175(8):2436-44.

21. Pratscher J, Vollmers J, Wiegand S, Dumont MG, Kaster A-K. Unravelling the identity, metabolic potential and global biogeography of the atmospheric methane-oxidizing upland soil sluster $\alpha$. Environmental Microbiology. 2018;20(3):1016-29.

709 22. Mobarry BK, Wagner M, Urbain V, Rittmann BE, Stahl DA. Phylogenetic probes for analyzing abundance and spatial organization of nitrifying bacteria. Applied and environmental microbiology. 1996;62(6):2156-62.

23. Happel E, Bartl I, Voss M, Riemann L. Extensive nitrification and active ammonia oxidizers in two contrasting coastal systems of the Baltic Sea. Environmental Microbiology. 2018;20(8):2913-26.

24. Lösekann T, Knittel K, Nadalig T, Fuchs B, Niemann H, Boetius A, et al. Diversity and abundance of aerobic and anaerobic methane oxidizers at the Haakon Mosby Mud volcano, Barents Sea. Applied and environmental microbiology. 2007;73(10):3348-62.

718 Reports. 2009;1(5):336-46.

719 26. Bellucci M, Curtis TP. Chapter Eleven - Ammonia-oxidizing bacteria in wastewater. In: Klotz MG, 720 Stein LY, editors. Methods in Enzymology. 496: Academic Press; 2011. p. 269-86.

721 27. Lawson CE, Lücker S. Complete ammonia oxidation: an important control on nitrification in 722 engineered ecosystems? Current Opinion in Biotechnology. 2018;50:158-65.

723 28. Stein LY. Insights into the physiology of ammonia-oxidizing microorganisms. Current opinion in 724 chemical biology. 2019;49:9-15.

725 29. Lehtovirta-Morley LE. Ammonia oxidation: Ecology, physiology, biochemistry and why they must all come together. FEMS Microbiology Letters. 2018;365(9):fny058.

727 30. Bédard C, Knowles R. Physiology, biochemistry, and specific inhibitors of $\mathrm{CH}_{4}, \mathrm{NH}_{4}{ }^{+}$, and $\mathrm{CO}$ oxidation by methanotrophs and nitrifiers. Microbiological Reviews. 1989;53(1):68-84.

31. Dalton H. Ammonia oxidation by the methane oxidising bacterium Methylococcus capsulatus strain Bath. Archives of Microbiology. 1977;114(3):273-9.

731 32. Poret-Peterson AT, Graham JE, Gulledge J, Klotz MG. Transcription of nitrification genes by the methane-oxidizing bacterium, Methylococcus capsulatus strain Bath. The Isme Journal. 2008;2:1213.

733 33. Hooper AB, Vannelli T, Bergmann DJ, Arciero DM. Enzymology of the oxidation of ammonia to 734 nitrite by bacteria. Anton Leeuw Int J Gen Mol Microbiol. 1997;71.

735 34. Zahn JA, DiSpirito AA. Membrane-associated methane monooxygenase from Methylococcus 736 capsulatus (Bath). Journal of Bacteriology. 1996;178(4):1018-29.

737 35. Holmes AJ, Costello A, Lidstrom ME, Murrell JC. Evidence that participate methane 738 monooxygenase and ammonia monooxygenase may be evolutionarily related. FEMS Microbiology 739 Letters. 1995;132(3):203-8. 
36. Khadka R, Clothier L, Wang L, Lim CK, Klotz MG, Dunfield PF. Evolutionary history of copper membrane monooxygenases. Frontiers in Microbiology. 2018;9(2493).

742 37. Ross MO, Rosenzweig AC. A tale of two methane monooxygenases. Journal of biological inorganic chemistry : JBIC : a publication of the Society of Biological Inorganic Chemistry. 2017;22(2-3):307-19. 38. Lund J, Woodland MP, Dalton H. Electron transfer reactions in the soluble methane monooxygenase of Methylococcus capsulatus (Bath). European Journal of Biochemistry. 1985;147(2):297305.

39. Hanson RS, Hanson TE. Methanotrophic bacteria. Microbiological Reviews. 1996;60(2):439-71.

40. Dedysh SN, Knief C, Dunfield PF. Methylocella species are facultatively methanotrophic. Journal of bacteriology. 2005;187(13):4665-70.

41. Hakemian AS, Rosenzweig AC. The biochemistry of methane oxidation. Annual Review of Biochemistry. 2007;76(1):223-41.

752 42. Suzuki I, Kwok S-C, Dular U. Competitive inhibition of ammonia oxidation in Nitrosomonas europaea by methane, carbon monoxide or methanol. FEBS Letters. 1976;72(1):117-20.

754 43. Keener WK, Arp DJ. Kinetic studies of ammonia monooxygenase inhibition in Nitrosomonas europaea by hydrocarbons and halogenated hydrocarbons in an optimized whole-cell assay. Applied and environmental microbiology. 1993;59(8):2501-10.

44. Hyman MR, Murton IB, Arp DJ. Interaction of ammonia monooxygenase from Nitrosomonas europaea with alkanes, alkenes, and alkynes. Applied and environmental microbiology. 1988;54(12):318790.

45. Hubley JH, Thomson AW, Wilkinson JF. Specific inhibitors of methane oxidation in Methylosinus trichosporium. Archives of Microbiology. 1975;102(1):199-202.

46. Prior SD, Dalton $\mathrm{H}$. The effect of copper ions on membrane content and methane monooxygenase activity in methanol-grown cells of Methylococcus capsulatus (Bath). Microbiology. 1985;131(1):155-63. 47. Pham MD, Lin YP, Van Vuong Q, Nagababu P, Chang BT, Ng KY, et al. Inactivation of the particulate methane monooxygenase (pMMO) in Methylococcus capsulatus (Bath) by acetylene. Biochimica et biophysica acta. 2015;1854(12):1842-52.

48. Arp DJ, Stein LY. Metabolism of inorganic N compounds by ammonia-oxidizing bacteria. Critical Reviews in Biochemistry and Molecular Biology. 2003;38(6):471-95. of C2 to C10 1-alkynes on ammonia oxidation in two Nitrososphaera species. Applied and environmental

772 50. Prior SD, Dalton H. Acetylene as a suicide substrate and active site probe for methane 773 monooxygenase from Methylococcus capsulatus (Bath). FEMS Microbiology Letters. 1985;29(1-2):105-9.

774 51. Lontoh S, DiSpirito AA, Krema CL, Whittaker MR, Hooper AB, Semrau JD. Differential inhibition in 775 vivo of ammonia monooxygenase, soluble methane monooxygenase and membrane-associated methane 776 monoxygenase by phenylacetylene. Environ Microbiol. 2000;2(5):485-94.

777 52. Fosdike WL, Smith TJ, Dalton H. Adventitious reactions of alkene monooxygenase reveal common 778 reaction pathways and component interactions among bacterial hydrocarbon oxygenases. The FEBS 779 Journal. 2005;272(11):2661-9.

780 53. Koops HP, Böttcher, B., Möller, U. C., Pommerening-Röser, A., Stehr, G. . Classification of eight 781 new species of ammonia-oxidizing bacteria: Nitrosomonas communis sp. nov., Nitrosomonas ureae sp. 
nov., Nitrosomonas aestuarii sp. nov., Nitrosomonas marina sp. nov., Nitrosomonas nitrosa sp. nov., Nitrosomonas eutropha sp. nov., Nitrosomonas oligotropha sp. nov. and Nitrosomonas halophila sp. nov. JGenMicrobiol. 1991;137:1689-99.

54. Kip N, Ouyang W, van Winden J, Raghoebarsing A, van Niftrik L, Pol A, et al. Detection, isolation, and characterization of acidophilic methanotrophs from Sphagnum mosses. Applied and environmental microbiology. 2011;77(16):5643-54.

788 55. Dedysh SN, Berestovskaya YY, Vasylieva LV, Belova SE, Khmelenina VN, Suzina NE, et al. Methylocella tundrae sp. nov., a novel methanotrophic bacterium from acidic tundra peatlands. International Journal of Systematic and Evolutionary Microbiology. 2004;54(1):151-6. oryzae Strain $\mathrm{C50C1}$ is a novel type Ib gammaproteobacterial methanotroph adapted to freshwater environments. mSphere. 2019;4(3):e00631-18.

57. Mundinger AB, Lawson CE, Jetten MSM, Koch H, Lücker S. Cultivation and transcriptional analysis of a canonical Nitrospira under stable growth conditions. Frontiers in Microbiology. 2019;10(1325).

58. Kits KD, Sedlacek CJ, Lebedeva EV, Han P, Bulaev A, Pjevac P, et al. Kinetic analysis of a complete nitrifier reveals an oligotrophic lifestyle. Nature. 2017;549:269.

59. Hogendoorn C, Pol A, Picone N, Cremers G, van Alen TA, Gagliano AL, et al. Hydrogen and carbon monoxide-utilizing Kyrpidia spormannii species from Pantelleria island, Italy. Frontiers in microbiology. 2020;11:951-.

60. Sakoula D, Koch H, Frank J, Jetten MSM, van Kessel MAHJ, Lücker S. Enrichment and physiological characterization of a novel comammox Nitrospira indicates ammonium inhibition of complete nitrification. The ISME Journal. 2020.

61. Taylor S, Ninjoor V, Dowd DM, Tappel AL. Cathepsin B2 measurement by sensitive fluorometric ammonia analysis. Analytical Biochemistry. 1974;60(1):153-62.

62. Griess P. Bemerkungen zu der Abhandlung der HH. Weselsky und Benedikt „Ueber einige

808 63. Miranda KM, Espey MG, Wink DA. A rapid, simple spectrophotometric method for simultaneous detection of nitrate and nitrite. Nitric Oxide. 2001;5(1):62-71.

810 64. Daims H. Use of fluorescence in situ hybridization and the daime image analysis program for the 811 cultivation-independent quantification of microorganisms in environmental and medical samples. Cold 812 Spring Harbor protocols. 2009;2009(7):pdb.prot5253.

813 65. Stoecker K, Dorninger C, Daims H, Wagner M. Double labeling of oligonucleotide probes for 814 fluorescence in situ hybridization (DOPE-FISH) improves signal intensity and increases rRNA accessibility. 815 Applied and environmental microbiology. 2010;76(3):922-6.

816 66. Slot JW, Geuze HJ. Cryosectioning and immunolabeling. Nat Protocols. 2007;2(10):2480-91.

817 67. Tokuyasu KT. A technique for ultracryotomy of cell suspensions and tissues. The Journal of Cell 818 Biology. 1973;57(2):551-65.

819 68. Liou W, Geuze HJ, Slot JW. Improving structural integrity of cryosections for immunogold labeling. 820 Histochemistry and Cell Biology. 1996;106(1):41-58.

821 69. Daims H, Lücker S, Wagner M. daime, a novel image analysis program for microbial ecology and 822 biofilm research. Environmental Microbiology. 2006;8(2):200-13. 
823 70. Daims H, Wagner M. Quantification of uncultured microorganisms by fluorescence microscopy

824 and digital image analysis. Applied microbiology and biotechnology. 2007;75(2):237-48.

825 71. Bushnell B. BBMap [Available from: https://jgi.doe.gov/data-and-tools/bbtools/.

826 72. Nurk S, Meleshko D, Korobeynikov A, Pevzner PA. metaSPAdes: a new versatile metagenomic 827 assembler. Genome Res. 2017.

828 73. Li H, Durbin R. Fast and accurate long-read alignment with Burrows-Wheeler transform. 829 Bioinformatics. 2010;26(5):589-95.

830 74. Li H, Handsaker B, Wysoker A, Fennell T, Ruan J, Homer N, et al. The Sequence Alignment/Map 831 format and SAMtools. Bioinformatics. 2009;25(16):2078-9.

832 75. Graham ED, Heidelberg JF, Tully BJ. BinSanity: unsupervised clustering of environmental microbial 833 assemblies using coverage and affinity propagation. PeerJ. 2017;5:e3035.

834 76. Lu YY, Chen T, Fuhrman JA, Sun F. COCACOLA: binning metagenomic contigs using sequence 835 COmposition, read CoverAge, CO-alignment and paired-end read LinkAge. Bioinformatics. 836 2017;33(6):791-8.

837 77. Alneberg J, Bjarnason BS, de Bruijn I, Schirmer M, Quick J, ljaz UZ, et al. Binning metagenomic 838 contigs by coverage and composition. Nature Methods. 2014;11(11):1144-6.

839 78. Wu YW, Simmons BA, Singer SW. MaxBin 2.0: an automated binning algorithm to recover 840 genomes from multiple metagenomic datasets. Bioinformatics. 2016;32(4):605-7.

841 79. Kang DD, Froula J, Egan R, Wang Z. MetaBAT, an efficient tool for accurately reconstructing single 842 genomes from complex microbial communities. PeerJ. 2015;3:e1165.

843 80. Sieber CMK, Probst AJ, Sharrar A, Thomas BC, Hess M, Tringe SG, et al. Recovery of genomes from 844 metagenomes via a dereplication, aggregation, and scoring strategy. bioRxiv. 2017.

845 81. Parks DH, Imelfort M, Skennerton CT, Hugenholtz P, Tyson GW. CheckM: assessing the quality of 846 microbial genomes recovered from isolates, single cells, and metagenomes. Genome Res. 847 2015;25(7):1043-55.

848 82. Parks DH, Chuvochina M, Waite DW, Rinke C, Skarshewski A, Chaumeil P-A, et al. A standardized 849 bacterial taxonomy based on genome phylogeny substantially revises the tree of life. Nature 850 Biotechnology. 2018;36:996.

851 83. Eren AM, Esen ÖC, Quince C, Vineis JH, Morrison HG, Sogin ML, et al. Anvi'o: an advanced analysis 852 and visualization platform for 'omics data. PeerJ. 2015;3:e1319.

853 84. Wick RR, Schultz MB, Zobel J, Holt KE. Bandage: interactive visualization of de novo genome 854 assemblies. Bioinformatics. 2015;31(20):3350-2.

855 85. Altschul SF, Gish W, Miller W, Myers EW, Lipman DJ. Basic local alignment search tool. Journal of 856 Molecular Biology. 1990;215(3):403-10.

857 86. Olm MR, Brown CT, Brooks B, Banfield JF. dRep: a tool for fast and accurate genomic comparisons 858 that enables improved genome recovery from metagenomes through de-replication. The ISME Journal. $8592017 ; 11(12): 2864-8$.

860 87. Seemann T. Prokka: rapid prokaryotic genome annotation. Bioinformatics. 2014;30(14):2068-9.

861 88. Hyatt D, Chen G-L, LoCascio PF, Land ML, Larimer FW, Hauser L. Prodigal: prokaryotic gene 862 recognition and translation initiation site identification. BMC Bioinformatics. 2010;11(1):119.

863 89. Eddy SR. Accelerated profile HMM searches. PLOS Computational Biology. 2011;7(10):e1002195. 
864 90. El-Gebali S, Mistry J, Bateman A, Eddy SR, Luciani A, Potter SC, et al. The Pfam protein families 865 database in 2019. Nucleic Acids Research. 2018;47(D1):D427-D32.

866 91. Camacho C, Coulouris G, Avagyan V, Ma N, Papadopoulos J, Bealer K, et al. BLAST+: architecture 867 and applications. BMC Bioinformatics. 2009;10(1):421.

868 92. Team R. Core R: A Language and Environment for Statistical Computing, Version 3.5. 3. Vienna: R 869 Foundation for Statistical Computing. 2019.

870 93. Wickham H, Averick M, Bryan J, Chang W, McGowan LDA, François R, et al. Welcome to the 871 Tidyverse. Journal of Open Source Software. 2019;4(43):1686.

872 94. Wright CL, Schatteman A, Crombie AT, Murrell JC, Lehtovirta-Morley LE. Inhibition of ammonia 873 monooxygenase from ammonia-oxidizing Archaea by linear and aromatic alkynes. Applied and 874 environmental microbiology. 2020;86(9):e02388-19.

875 95. Geets J, Boon N, Verstraete W. Strategies of aerobic ammonia-oxidizing bacteria for coping with 876 nutrient and oxygen fluctuations. FEMS Microbiology Ecology. 2006;58(1):1-13.

877 96. Kitmitto A, Myronova N, Basu P, Dalton H. Characterization and structural analysis of an active 878 particulate methane monooxygenase trimer from Methylococcus capsulatus (Bath). Biochemistry. 879 2005;44(33):10954-65.

880 97. Fiencke C, Bock E. Immunocytochemical localization of membrane-bound ammonia 881 monooxygenase in cells of ammonia oxidizing bacteria. Arch Microbiol. 2006;185(2):99-106.

882 98. Murray RGE, Watson SW. Structure of Nitrosocystis oceanus and comparison with Nitrosomonas 883 and Nitrobacter. Journal of Bacteriology. 1965;89(6):1594-609.

884 99. Smith U, Ribbons DW, Smith DS. The fine structure of Methylococcus capsulatus. Tissue and Cell. 885 1970;2(4):513-20.

886 100. Zhou Z, Liu Y, Pan J, Cron BR, Toner BM, Anantharaman K, et al. Gammaproteobacteria mediating 887 utilization of methyl-, sulfur- and petroleum organic compounds in deep ocean hydrothermal plumes. The 888 ISME Journal. 2020;14(12):3136-48.

889 101. Coleman NV, Le NB, Ly MA, Ogawa HE, McCarl V, Wilson NL, et al. Hydrocarbon monooxygenase 890 in Mycobacterium: recombinant expression of a member of the ammonia monooxygenase superfamily. 891 The ISME Journal. 2012;6(1):171-82.

892 102. Palomo A, Pedersen AG, Fowler SJ, Dechesne A, Sicheritz-Pontén T, Smets BF. Comparative 893 genomics sheds light on niche differentiation and the evolutionary history of comammox Nitrospira. The 894 ISME Journal. 2018;12(7):1779-93.

895 103. Shah VK, Davis LC, Brill WJ. Nitrogenase: VI. Acetylene reduction assay: Dependence of nitrogen 896 fixation estimates on component ratio and acetylene concentration. Biochimica et Biophysica Acta (BBA) 897 - Enzymology. 1975;384(2):353-9.

898 104. Whidbey C, Wright AT. Activity-based protein profiling-Enabling mltimodal fnctional studies of 899 microbial communities. In: Cravatt BF, Hsu K-L, Weerapana E, editors. Activity-Based Protein Profiling.

900 Cham: Springer International Publishing; 2019. p. 1-21.

901 105. Stein LY, Arp DJ, Hyman MR. Regulation of the synthesis and activity of ammonia monooxygenase 902 in Nitrosomonas europaea by altering $\mathrm{pH}$ to affect $\mathrm{NH}_{3}$ availability. Applied and environmental 903 microbiology. 1997;63(11):4588-92. 
904 106. Pjevac P, Schauberger C, Poghosyan L, Herbold CW, van Kessel MAHJ, Daebeler A, et al. AmoA905 targeted polymerase chain reaction primers for the specific detection and quantification of comammox 906 Nitrospira in the environment. Frontiers in Microbiology. 2017;8(1508).

907 107. Pinto AJ, Marcus DN, ljaz UZ, Bautista-de Lose Santos QM, Dick GJ, Raskin L. Metagenomic 908 evidence for the presence of comammox Nitrospira-like bacteria in a drinking water system. mSphere. 909 2016;1(1).

910 108. Palomo A, Jane Fowler S, Gülay A, Rasmussen S, Sicheritz-Ponten T, Smets BF. Metagenomic 911 analysis of rapid gravity sand filter microbial communities suggests novel physiology of Nitrospira spp. 9122016.

913 109. Bartelme R, McLellan S, Newton R. Freshwater recirculating aquaculture system operations drive 914 biofilter bacterial community shifts around a stable nitrifying consortium of ammonia-oxidizing archaea 915 and comammox Nitrospira. Frontiers in Microbiology. 2017;8(101).

916 110. Wang J-G, Xia F, Zeleke J, Zou B, Rhee S-K, Quan Z-X. An improved protocol with a highly 917 degenerate primer targeting copper-containing membrane-bound monooxygenase (CuMMO) genes for 918 community analysis of methane- and ammonia-oxidizing bacteria. FEMS Microbiology Ecology. 2016.

919 111. Rotthauwe JH, Witzel KP, Liesack W. The ammonia monooxygenase structural gene amoA as a 920 functional marker: molecular fine-scale analysis of natural ammonia-oxidizing populations. Applied and 921 environmental microbiology. 1997;63(12):4704-12.

922 112. Ghashghavi M, Jetten MSM, Lüke C. Survey of methanotrophic diversity in various ecosystems by 923 degenerate methane monooxygenase gene primers. AMB Express. 2017;7(1):162-.

924 113. Fujitani $\mathrm{H}$, Kumagai A, Ushiki N, Momiuchi K, Tsuneda S. Selective isolation of ammonia-oxidizing 925 bacteria from autotrophic nitrifying granules by applying cell-sorting and sub-culturing of microcolonies. 926 Frontiers in microbiology. 2015;6:1159-.

927 114. Morgan-Sagastume F, Larsen P, Nielsen JL, Nielsen PH. Characterization of the loosely attached 928 fraction of activated sludge bacteria. Water Research. 2008;42(4):843-54.

929 115. Mcllroy SJ, Albertsen M, Andresen EK, Saunders AM, Kristiansen R, Stokholm-Bjerregaard M, et 930 al. 'Candidatus Competibacter'-lineage genomes retrieved from metagenomes reveal functional 931 metabolic diversity. The Isme Journal. 2013;8:613.

932 116. Mcllroy SJ, Nittami T, Kanai E, Fukuda J, Saunders AM, Nielsen PH. Re-appraisal of the phylogeny 933 and fluorescence in situ hybridization probes for the analysis of the Competibacteraceae in wastewater 934 treatment systems. Environmental Microbiology Reports. 2015;7(2):166-74. 


\section{Figure legends}

937 Figure 1. Inhibition of the ammonia oxidation activity of the comammox organism Ca. N. kreftii by 1,70D. (A)

938 Ammonium consumption and (B) nitrate production in the presence of $350 \mu \mathrm{M} \mathrm{NH}_{4}{ }^{+}$with and without the

939 addition of $100 \mu \mathrm{M}$ 1,70D or ATU. (C) Nitrite consumption and (D) nitrate production in the presence of $20 \mu \mathrm{M}$

$940 \mathrm{NO}_{2}{ }^{-}$with and without the addition of $100 \mu \mathrm{M}$ 1,70D or ATU. Concentrations of the substrates ammonium (A)

941 and nitrite $(C)$ are indicated by solid, produced nitrate $(B, D)$ by open symbols. Data points represent the mean

942 of technical triplicates, error bars the standard deviations between three biological replicates.

944 Figure 2. ABPP-based fluorescent labelling of catalytically active ammonia-oxidizing prokaryotes. (A, C, E, F) 945 1,70D pre-incubated and (B, D) 1,70D untreated (control) cells of (A, B) Nitrosomonas europaea, (C, D) 946 Nitrospira inopinata, (E) Nitrosocosmicus franklandus, (F) Nitrospira moscoviensis. Cells are stained with the 947 AMO/MMO-labelling protocol (green) and FISH probes specific for (A, B) AOB (Nso190, Neuo653, Nso1225; 948 red), (C, D, F) Nitrospira (Ntspa662, Ntspa712; red), (E) AOA (Arch915; red) and (A, B, F) all bacteria (EUB338mix; 949 blue).

Figure 3. ABPP-based fluorescent labelling of catalytically active methane oxidizing bacteria. $(A, C, E, G) 1,7 O D$

952 pre-incubated and (B, D, F, H) 1,70D untreated (control) cells of (A, B) Methylotetracoccus oryzae, (C, D)

953 Methylosinus sporium M29, (E, F) Methylacidiphilum fumariolicum SolV, (G, H) Methylocella tundrae. Cells are

954 stained with the AMO/MMO-labelling protocol (green) and FISH probes specific for (A, B)

955 Gammaproteobacteria (Gam42a; red), (C, D, G, H) Alphaproteobacteria (Alf0001b, Alf0968; red), (E, F)

956 Verrucomicrobia (EUB338 III; red), and (A-H) all bacteria (EUB338mix).

Figure 4. Correlation of the $\mathrm{AMO} / \mathrm{MMO}$-derived fluorescent signal intensity and staining efficiency with the 959 growth stage of Nitrosomonas europaea. (A) Ammonia consumption and nitrite production activity observed

960 in the culture during the incubation period. (B) Growth of the bacterial culture as indicated by protein content 961 and its relation to the relative intensity of the fluorescent signal observed, as well as the biovolume fraction of 962 the culture stained by the AMO/MMO-based protocol. Bacterial growth data are fitted to a Hill equation. Error 963 bars represent standard deviation of technical triplicates of protein measurements.

965 Figure 5. Subcellular localization of the AMO/MMO-derived fluorescent signal using deconvolution confocal 966 microscopy. Cells of (A) Nitrosomonas europaea, (B) Methylotetracoccus oryzae and (C) Methylocella tundrae, 967 are stained with the fluorescent AMO/MMO protocol (green) and specific (A) AOB (Nso190, Neuo653, 
Nso1225; red), (B) gammaproteobacterial (Gam42a; red) or (C) alphaproteobacterial (Alf0001b, Alf0968; red) FISH probes.

Figure 6. Immuno-gold labelling of active AMO/MMO enzymes using transmission electron microscopy. (A, D)

972 1,7OD untreated (control) and (B, C, E, F) 1,7OD pre-incubated cells of (A-C) Nitrosomonas europaea and (D-F)

973 Methylotetracoccus oryzae. Scale bars correspond to $0.5 \mu \mathrm{m}$.

975 Figure 7. ABPP-based fluorescent labelling of catalytically active ammonia-oxidizing bacteria present in 976 complex microbial communities. (A, C) 1,70D pre-incubated and (B, D) 1,70D untreated (control) cells of (A, B) 977 a Ca. N. nitrosa and Ca. N. nitrificans and (C, D) a nitrifying enrichment culture. Cells are stained with the 978 AMO/MMO-labelling protocol (green signal) and FISH probes specific for Nitrospira (Ntspa662, Ntspa712; 979 shown in red [A, B] and in blue [C, D]), (C, D) AOB (Nso190, Neu653, Nso1225; in red) and (A, B) all bacteria 980 (EUB338mix; in blue).

982 Figure 8. Normalized and relative abundances of (A) $a m o A$ and (B) rpoB genes in metagenomes of untreated, 983 controls, and sorted samples. The top row shows the normalized read abundances in units of Reads Per Kilobase gene length per Million mapped reads (RPKM) as symbols colored by taxonomic affiliation, and the total number of reads mapped to the co-assembly as gray bars. The same gene for the taxa of interest, Nitrosomonas and comammox Nitrospira, are emphasized and connected with lines. The bottom shows the same RPKM data as the top row as a proportion of the total RPKM for the respective gene in the metagenomic sample, with each individual colored box representing the relative abundance of a single gene from the coassembly. The final treatment preceding the CUAAC reaction is indicated in bold along with the controls for the inactivation and dyeing, and the final sorted sample.

Figure 9. Differential coverage plot showing the abundance of contigs in the untreated activated sludge sample

993 in comparison to the ABPP labelled and sorted sample. Each circle represents a metagenomic contig, the circle

994 size corresponds to its length. The five most abundant MAGs in the ABPP-positive and sorted sample are 995 indicated by color, while the red filling indicates contigs on which genes encoding CuMMO (AMO or MMO) 996 subunits have been detected. 
bioRxiv preprint doi: https://doi.org/10.1101/2021.01.14.426632; this version posted January 14,2021 . The copyright holder for this preprint (which was not certified by peer review) is the author/funder, who has granted bioRxiv a license to display the preprint in perpetuity. It is made available under aCC-BY-NC 4.0 International license.

997
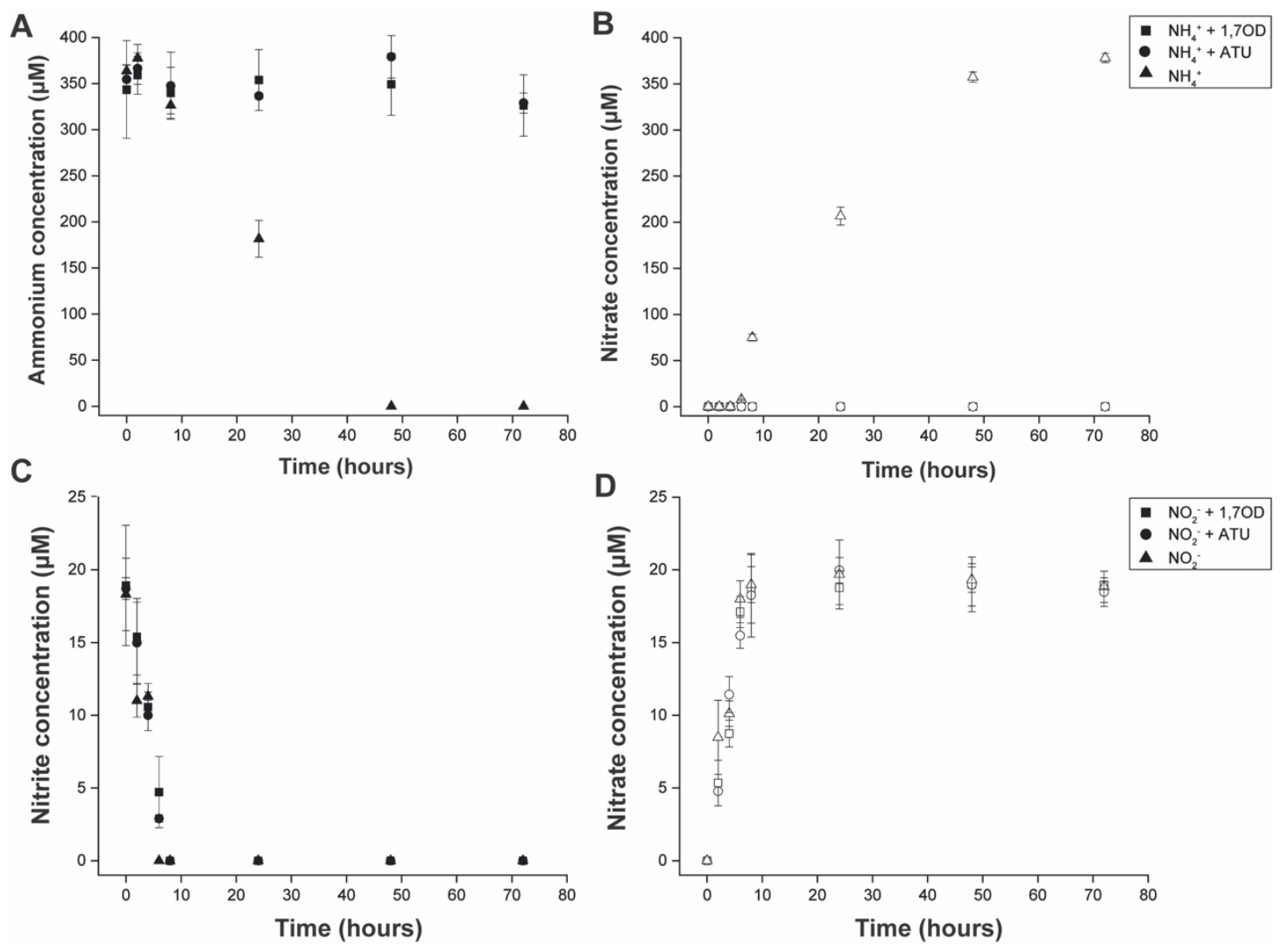

998

Figure 1. 
bioRxiv preprint doi: https://doi.org/10.1101/2021.01.14.426632; this version posted January 14, 2021. The copyright holder for this preprint (which was not certified by peer review) is the author/funder, who has granted bioRxiv a license to display the preprint in perpetuity. It is made available under aCC-BY-NC 4.0 International license.
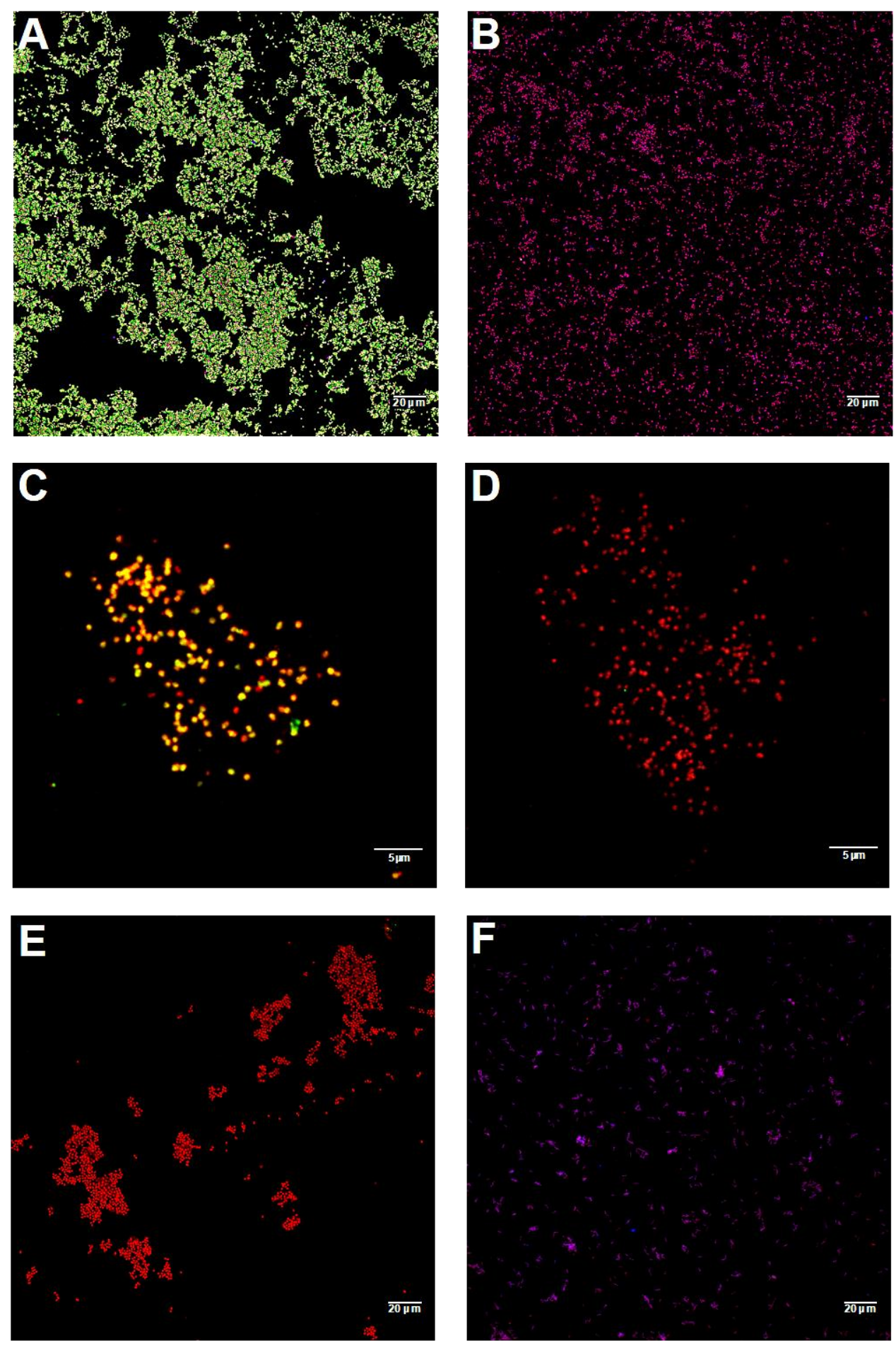

1000 Figure 2. 
bioRxiv preprint doi: https://doi.org/10.1101/2021.01.14.426632; this version posted January 14, 2021. The copyright holder for this preprint (which was not certified by peer review) is the author/funder, who has granted bioRxiv a license to display the preprint in perpetuity. It is made available under aCC-BY-NC 4.0 International license.
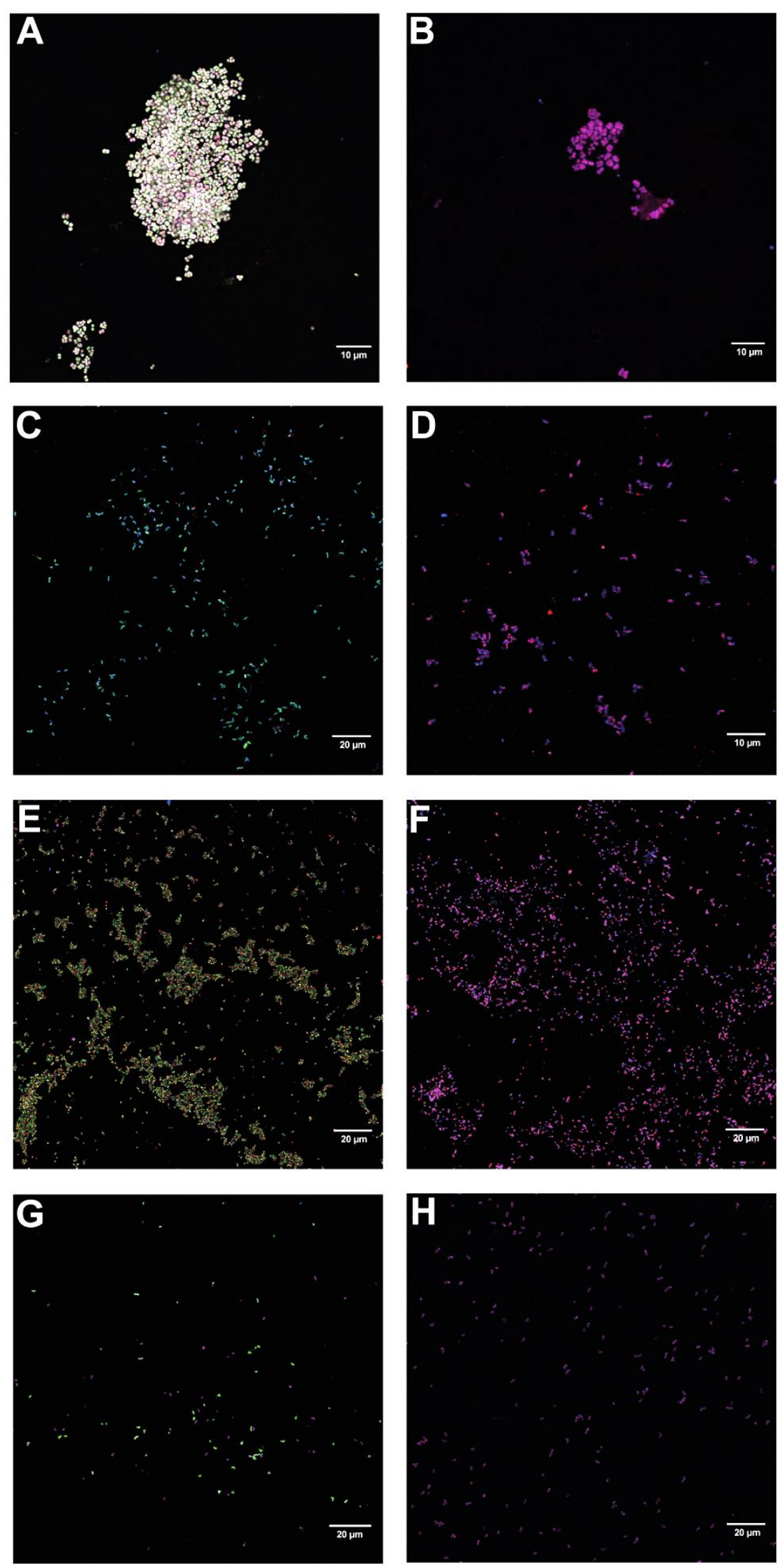

1002 Figure 3. 
bioRxiv preprint doi: https://doi.org/10.1101/2021.01.14.426632; this version posted January 14, 2021. The copyright holder for this preprint (which was not certified by peer review) is the author/funder, who has granted bioRxiv a license to display the preprint in perpetuity. It is made available under aCC-BY-NC 4.0 International license.

1003

A

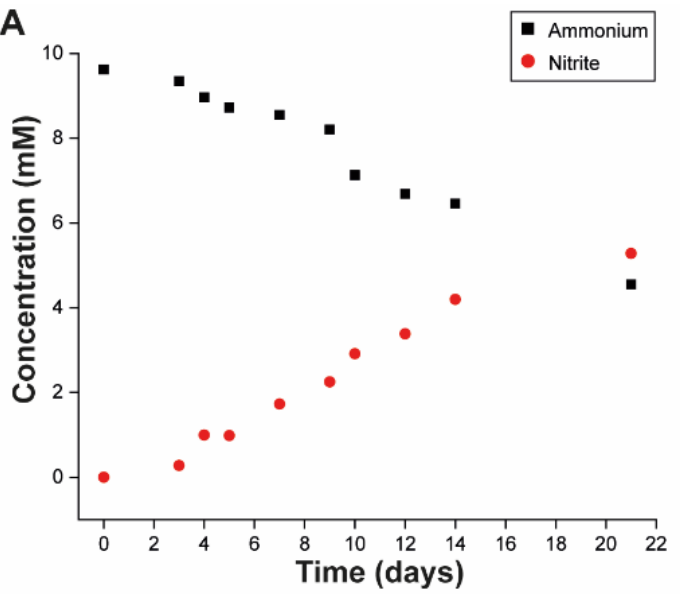

B

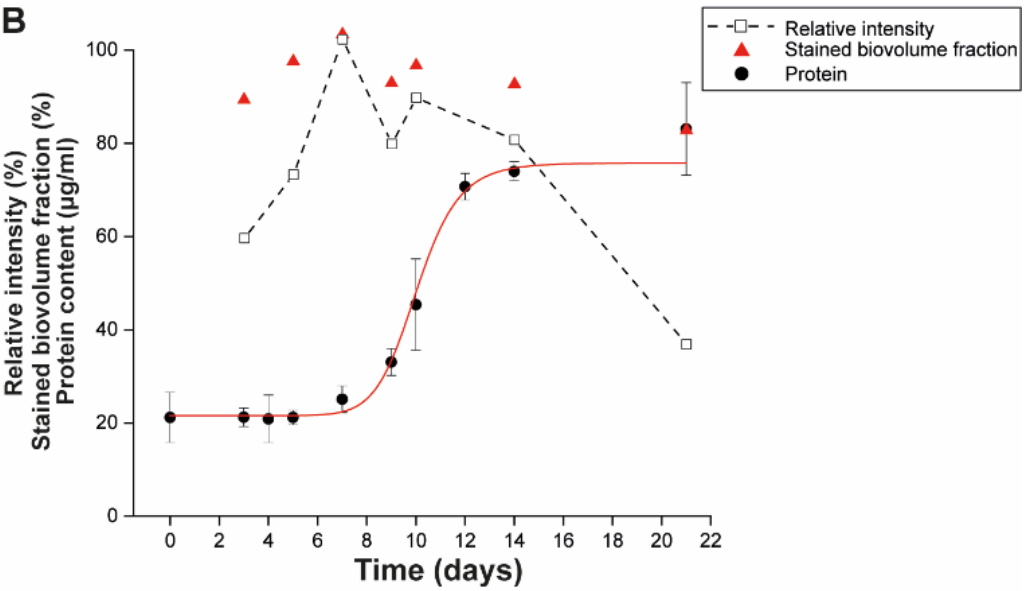

1004 Figure 4.

1005

1006

1007

1008

1009

1010
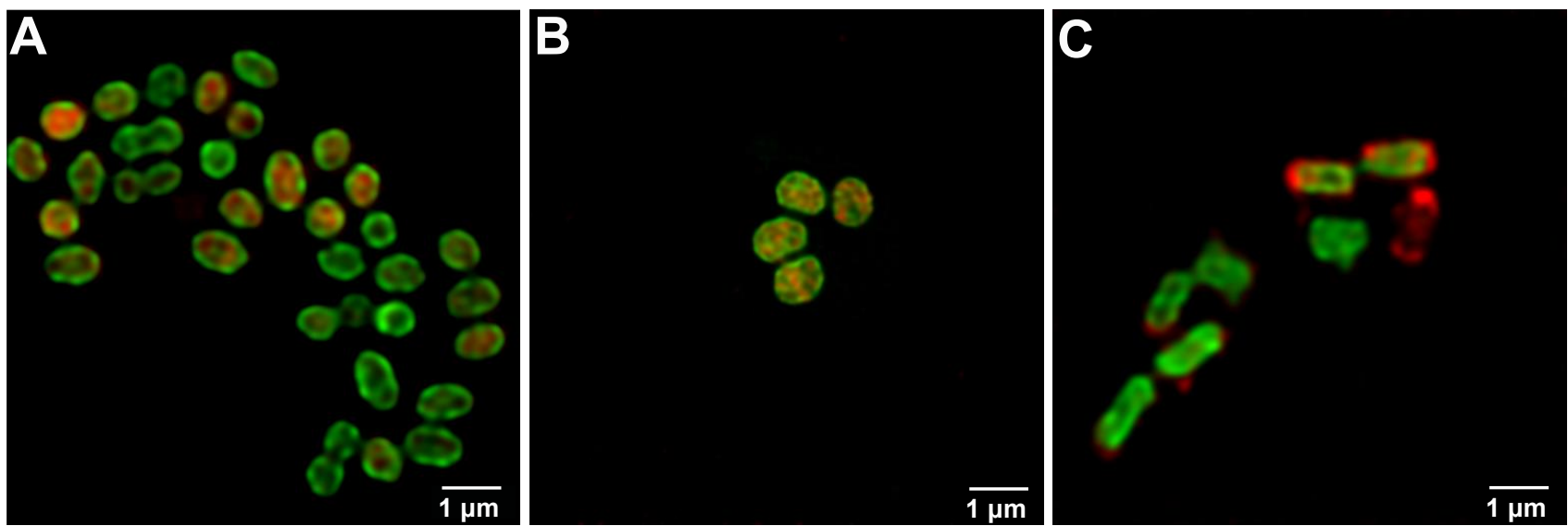

Figure 5. 
bioRxiv preprint doi: https://doi.org/10.1101/2021.01.14.426632; this version posted January 14,2021 . The copyright holder for this preprint (which was not certified by peer review) is the author/funder, who has granted bioRxiv a license to display the preprint in perpetuity. It is made available under aCC-BY-NC 4.0 International license.
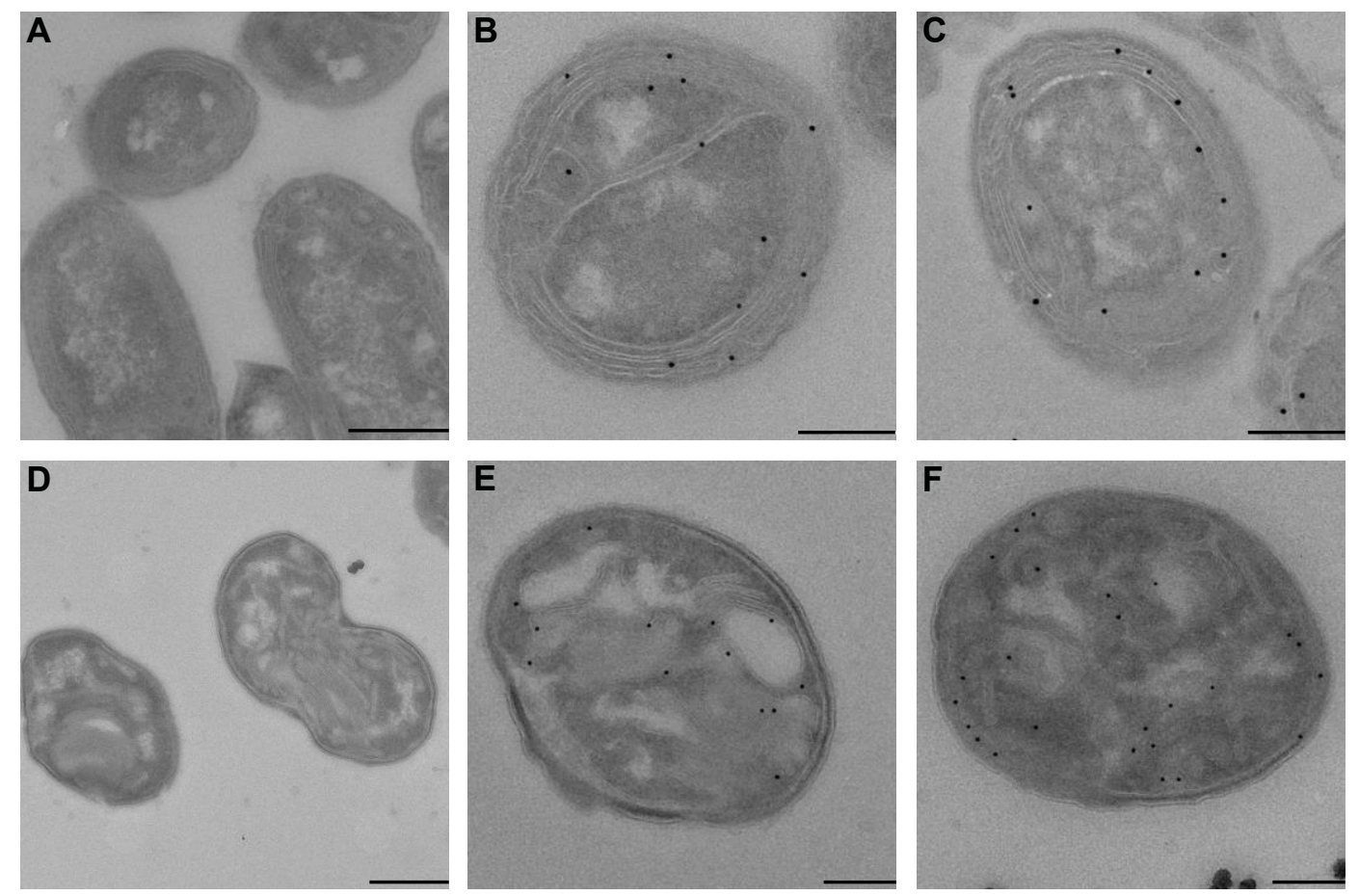

1012

1013 Figure 6. 
bioRxiv preprint doi: https://doi.org/10.1101/2021.01.14.426632; this version posted January 14, 2021. The copyright holder for this preprint (which was not certified by peer review) is the author/funder, who has granted bioRxiv a license to display the preprint in perpetuity. It is made available under aCC-BY-NC 4.0 International license.
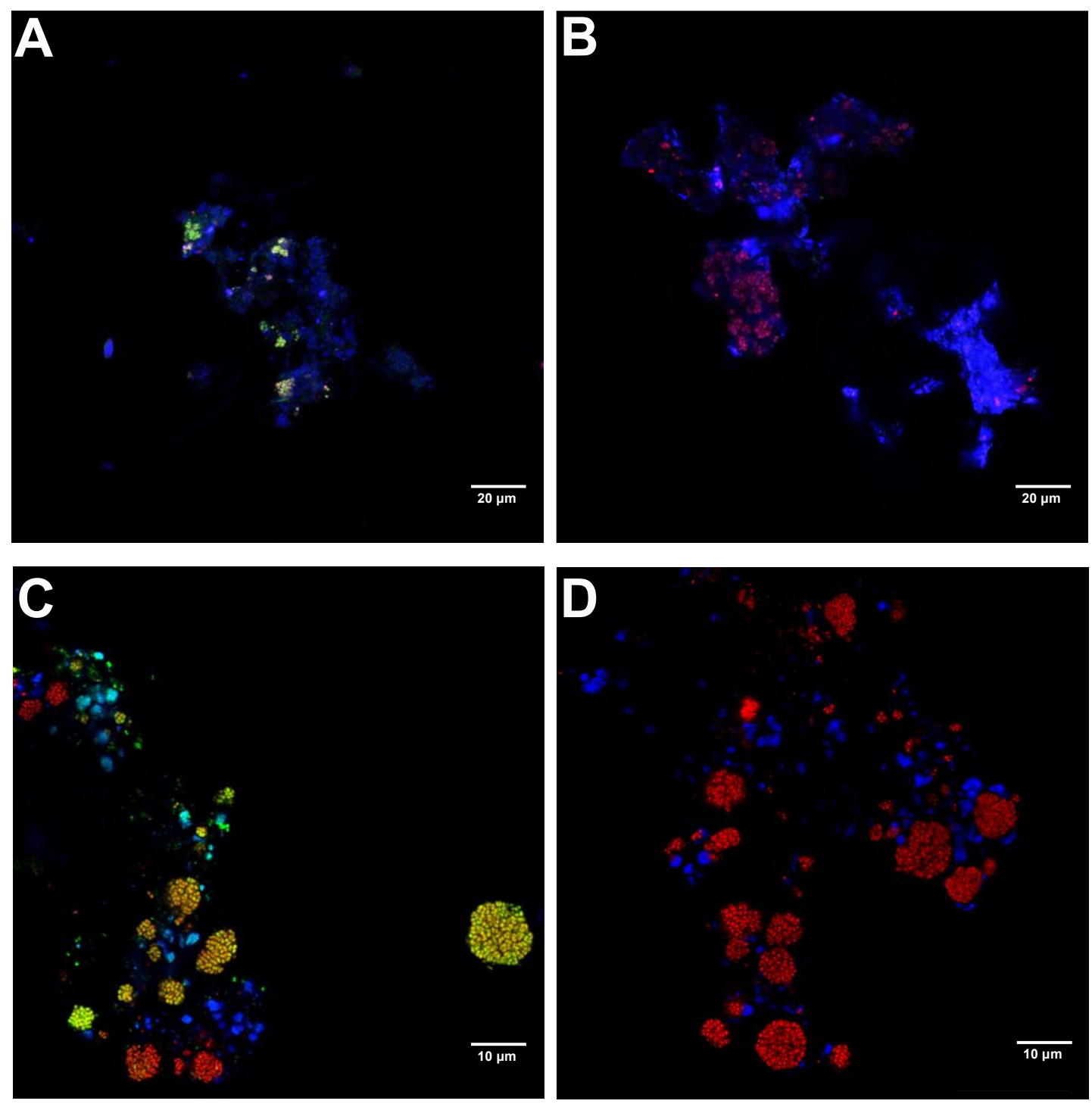

1014

1015 Figure 7. 
bioRxiv preprint doi: https://doi.org/10.1101/2021.01.14.426632; this version posted January 14,2021. The copyright holder for this preprint (which was not certified by peer review) is the author/funder, who has granted bioRxiv a license to display the preprint in perpetuity. It is made available under aCC-BY-NC 4.0 International license.

a
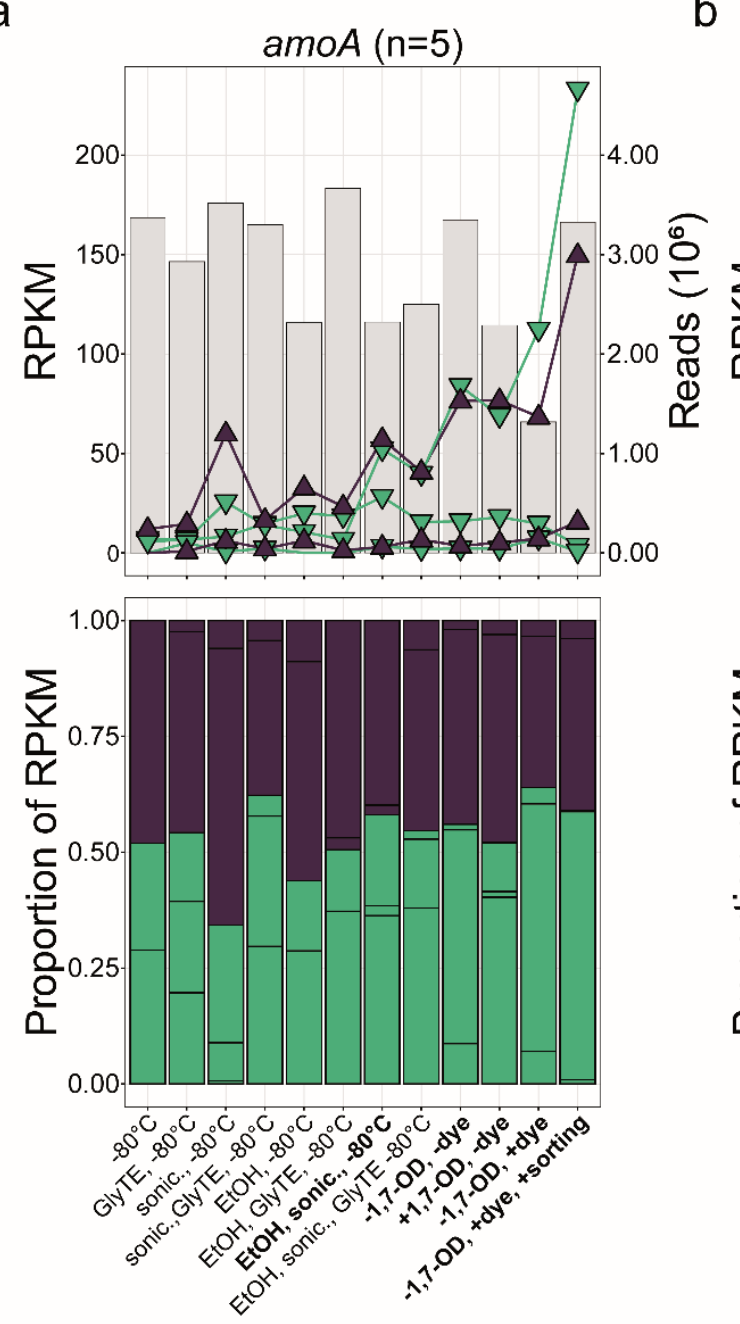

Gene affiliation b
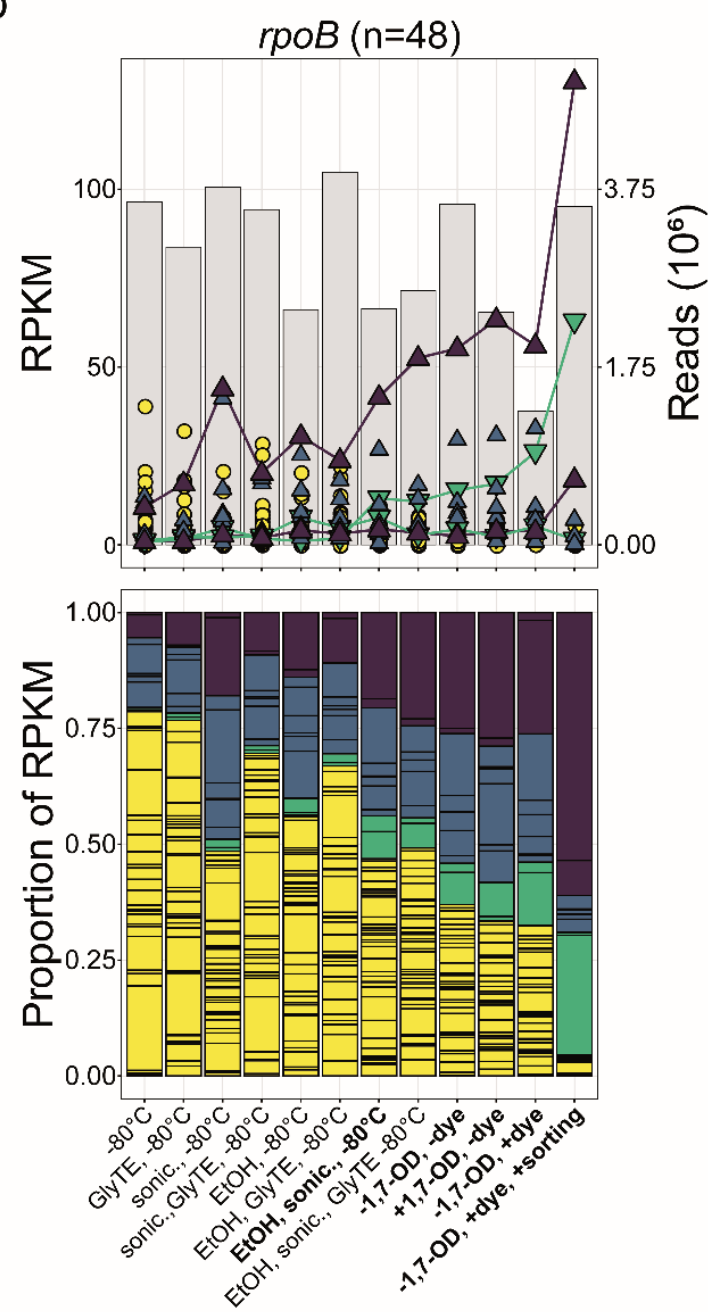
Canonical
Nitrospira $\nabla$ Nitrosomonas $\bigcirc$ Other 
bioRxiv preprint doi: https://doi.org/10.1101/2021.01.14.426632; this version posted January 14,2021 . The copyright holder for this preprint (which was not certified by peer review) is the author/funder, who has granted bioRxiv a license to display the preprint in perpetuity. It is made available under aCC-BY-NC 4.0 International license.

1018

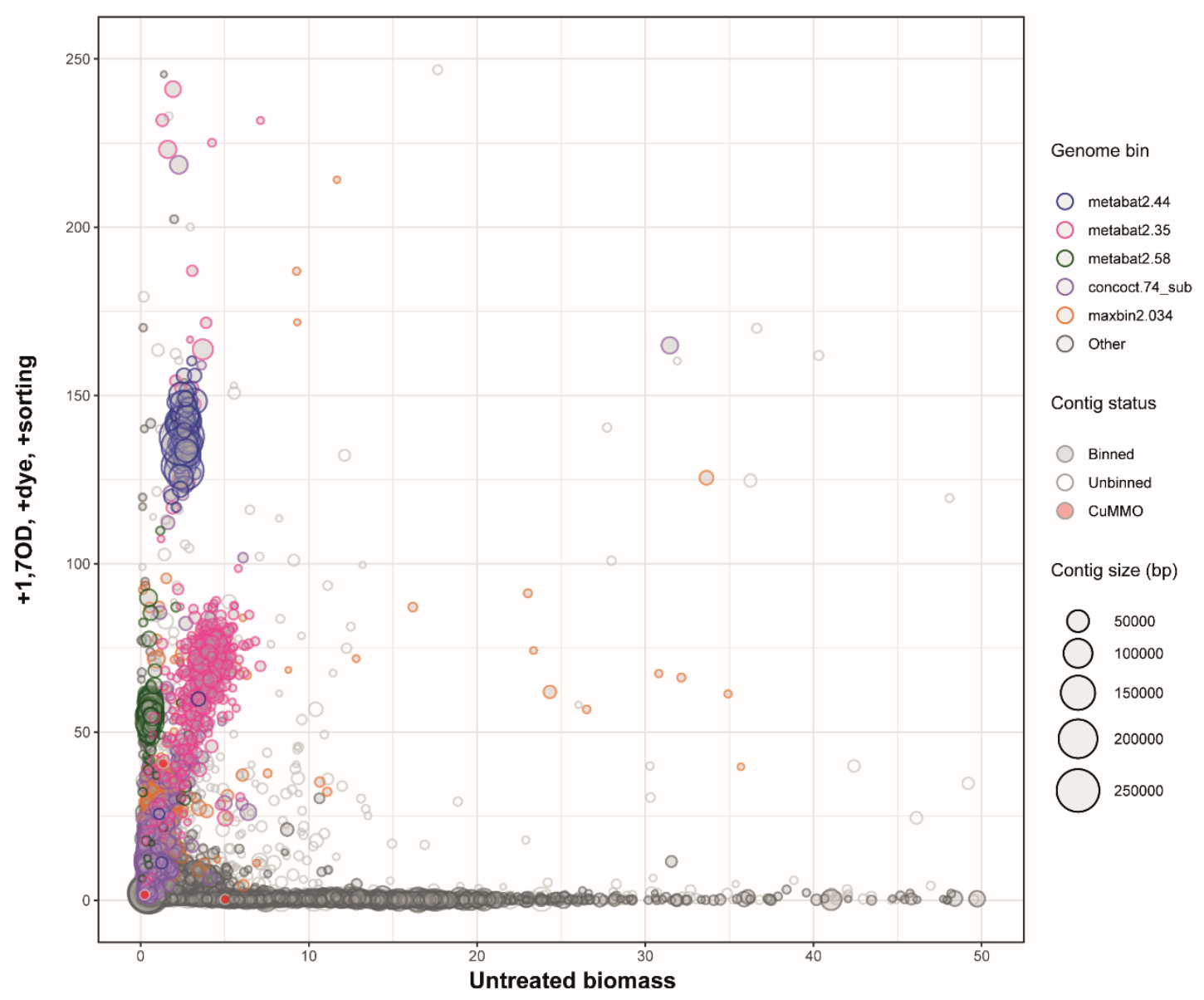

1019 Figure 9. 